\title{
On a Coverage Process Ranging from the Boolean Model to the Poisson Voronoi Tessellation \\ With Applications to Wireless Communications
}

François Baccelli — Bartłomiej Błaszczyszyn

$\mathbf{N}^{\circ} 4019$

Octobre 2000

THÈME 1 



\title{
On a Coverage Process Ranging from the Boolean Model to the Poisson Voronoi Tessellation With Applications to Wireless Communications
}

\author{
François Baccelli*, Bartłomiej Błaszczyszyn ${ }^{\dagger}$ \\ Thème 1 - Réseaux et systèmes \\ Projet MCR
}

Rapport de recherche $n^{\circ} 4019$ - Octobre 2000 - 38 pages

\begin{abstract}
We define and analyze a random coverage process of the $d$-dimensional Euclidean space which allows one to describe a continuous spectrum that ranges from the Boolean model to the Poisson-Voronoi tessellation to the Johnson-Mehl model. Like for the Boolean model, the minimal stochastic setting consists of a Poisson point process on this Euclidean space and a sequence of real valued random variables considered as marks of this point process. In this coverage process, the cell attached to a point is defined as the region of the space where the effect of the mark of this point exceeds an affine function of the cumulated effect of all marks. This cumulated effect is defined as the shot noise process associated with the marked point process.

In addition to analyzing and visualizing this continuum, we study various basic properties of the coverage process such as the probability that a point or a pair of points be covered by a typical cell. We also determine the distribution of the number of cells which cover a given point, and show how to provide deterministic bounds on this number. Finally, we also analyze convergence properties of the coverage process using the framework of closed sets, and its differentiability properties using perturbation analysis. Our results require a pathwise continuity property for the shot noise process for which we provide sufficient conditions.

The model in question stems from wireless communications where several antennas share the same (or different but interfering) channel(s). In this case, the area where the signal of a given antenna can be received is the area where the signal to interference ratio is large enough. We describe this class of problems in detail in the paper. The obtained results allow one to compute quantities of practical interest within this setting: for instance the outage probability is obtained as the complement of the volume fraction; the law of the number of cells covering a point allows one to characterize handover strategies etc.

* ENS/INRIA, 45 rue d'Ulm, 75005 Paris; e-mail: Francois.Baccelli@ens.fr. This research was partially funded by the France Télécom CTI grant N 198 E 2220041611012 and by the RNRT Georges project

$\dagger$ Mathematical Institute, University of Wrocław pl. Grunwaldzki 2/4, 50-384 Wrocław, Poland; e-mail: blaszcz@math.uni.wroc.pl. This research was done while the author was visiting ENS in Paris and was funded by the France Télécom CTI grant N 198 E 2220041611012
\end{abstract}


Key-words: Shot noise, Boolean model, Voronoi tessellation, coverage process, point process, Poisson process, random closed set, perturbation analysis, wireless communication, signal to interference ratio, CDMA protocol. 


\title{
Sur un processus de couverture contenant le modèle booléen et la mosaïque de Poisson-Voronoi
}

\author{
et ses applications aux communications sans fil
}

Résumé : Nous définissons et analysons un processus de couverture aléatoire sur l'espace euclidien de dimension $d$, qui permet de décrire un continuum allant du modèle booléen à la mosaïque de Voronoi en passant par le modèle de Johnson-Mehl. Comme pour le modèle booléen, les données minimales pour définir un tel processus sont un processus ponctuel de Poisson sur cet espace euclidien et une suite de variables aléatoires à valeurs réelles. La cellule attachée à un point est définie comme la région du plan où l'effet de la marque de ce point dépasse une fonction affine de l'effet cumulé de toutes les marques du processus ponctuel. Cet effet cumulé est par définition le processus de shot noise associé au processus ponctuel.

Le continuum de processus de couverture est alors obtenu en faisant varier les paramètres des marques. En plus de l'analyse et de la visualisation de ce continuum, nous étudions plusieurs propriétés fondamentales de ce processus de couverture, comme la probabilité de couverture d'un point ou d'une paire de points par une cellule typique, ou encore comme la distribution du nombre de cellules qui couvrent un point donné. Nous étudions aussi certaines propriétés de convergence de ce processus au moyen du formalisme des fermés aléatoires, et certaines questions de différentiabilité par l'analyse des perturbations. Ces résutats reposent sur une propriété de continuité trajectorielle du processus de shot noise pour laquelle nous donnons des conditions suffisantes.

Ce modèle est issu des communications sans fil où plusieurs antennes utilisent les mêmes canaux de fréquences, ou encore des canaux sujets à des interférences. Dans ce cadre, le domaine où une antenne donnée peut être reçue est celui où le rapport signal sur bruit (ou signal sur interférence) est plus grand qu'un certain seuil. Nous décrivons ces motivations en détail dans l'article. Les analyses mathématiques permettent de calculer certaines caractéristiques d'intérêt pratique dans ce cadre, comme la probabilité de non connexion; la loi du nombre de cellules couvrant un point donné permet quant à elle de caractériser certaines propriétés du handover.

Mots-clés : Shot noise, Modèle booléen, mosaïque de Voronoi, processus de couverture, processus ponctuel, processus de Poisson, fermé aléatoire, analyse des perturbations, communication sans fil, rapport signal sur bruit, protocole CDMA. 


\section{Introduction}

Consider a marked point process $\Phi$ in the $d$-dimensional Euclidean space $\mathbb{R}^{d}$, where marks are real valued. Assume we have a positive, decreasing function $l$, called the attenuation function, defined on $\mathbb{R}^{d}$ (in the shot-noise literature it is called the response function). Consider the effect of the mark $S$ of some point $X$ of $\Phi$ on location $x$ in the space, to be the product of $S$ and the attenuation along the path from $X$ to $x$, namely $S l(x-X)$. Define the cell associated to point $X$ as the region of the plane where the ratio of the effect of mark $S$ over the sum of the effects of all marks (sometimes referred to as the shot-noise) and of some external noise, is larger than some threshold $\theta$, which is another mark attached to location $X$.

What is then the shape of such a cell? What interactions exist between adjacent or remote cells? Under natural stationarity and ergodicity assumptions, what is the proportion of the space which belongs to exactly $k$ cells? Is it possible to have coverage of $x$ by arbitrarily many cells? These are the main questions which will be addressed in the present paper. For computational results, we primarily concentrate on the Poisson point process case with independent marking. Certain existence results hold true for more general point processes.

To the best of our knowledge, this model is new in the stochastic geometry setting. As indicated in the abstract, we think it is nevertheless an important model in that it contains several basic models of this field as particular limiting cases, including the Voronoi tessellation, the Boolean model and the Johnson-Mehl model, and in that it also provides a parametric continuum linking all of them.

The model in question stems from the analysis of wireless communication systems where several antennas share the same (or different but interfering) channel(s), and where the good reception of the signal emitted by an antenna depends on the signal to noise or signal to interference ratio. This is for instance the case for the CDMA (Code Division Multiple Access) technology which is one of the basic schemes of wireless communications.

Within this setting, one should interpret points of $\Phi$ as describing locations of either fixed or mobile base-transceiver-stations and marks $(S, \theta)$ as representing pilot-signal-levels and pilotto-noise-ratios respectively. The cell associated with an antenna is then the part of the plane one user can extract the signal emitted by this antenna from the noise due to the interferences with signals emitted by other antennas or other users.

The paper is structured as follows: the model is described in Section 2. We first describe the generic stochastic geometry model and then consider several incarnations of this, all motivated by wireless communications. The characteristics of typical cells are studied in Section 4 . The law of the coverage of a given location of the plane is considered in Section 5, where we study in particular the so called volume fraction. Section 6 concentrates on the parametric continuum alluded to above, and elucidates the relationship between our model and those indicated above. We illustrate this by simulations showing the continuous transformation from a Boolean model to a Voronoi or Johnson-Mehl model and by various convergence results and perturbation expansions.

\section{Description of the model}

\subsection{Notation}

In what follows, $B(x, r)$, (resp. $B^{o}(x, r)$ ) will denote the closed (resp. open) ball centered at $x$ and of radius $r ; \mathbf{b}_{d}$ will denote the volume of the unit ball in $\mathbb{R}^{d}$ and $\nu_{d}(\cdot)$ the $d$-dimensional 
Lebesgue's measure. Moreover, we denote by $\stackrel{\circ}{B}$ the interior of set $B \subset \mathbb{R}^{d}$, by $A \oplus B=\{y+x$ : $y \in A, x \in B\}$ the Minkowski sum of sets $A$ and $B$, and by $\check{B}$ that set $\left\{-y \in \mathbb{R}^{d}: y \in B\right\}$. Let also $\delta_{z}$ be the Dirac measure at $z$; i.e., $\delta_{z}(A)=1$, if $z \in A$ and 0 otherwise. Finally, let $\mathcal{F}_{K}=\left\{F \subset \mathbb{R}^{d}\right.$ closed $\left.: F \cap K \neq \emptyset\right\}$. We recall that the family of sets $\mathcal{F}_{K}$, where $K$ ranges over compact sets, generates a $\sigma$-algebra which allows one to consider closed subsets of $\mathbb{R}^{d}$ as random variables (see Section 2-1, p. 27 in [12]). Moreover we will consider the compact topology on the space of closed sets (see Section 1-2, p. 3 in [12]).

\subsection{Generic stochastic geometry model}

Let $\Phi=\left\{\left(X_{i}, Z_{i}\right)\right\}$ be a marked point process on the $d$-dimensional Euclidean space $\mathbb{R}^{d}$, where $\left\{X_{i}\right\}$ denotes the locations of points, and where the marks $Z_{i}=\left(S_{i}, A_{i}\right)$ are such that $S_{i}$ belong to some metric space $\mathbb{D}$ and $A_{i}=\left(a_{i}, b_{i}, c_{i}\right) \in(\mathbb{R})^{3}$.

In addition to this marked point process, the model is based on a function $L: \mathbb{D} \times \mathbb{R}^{d} \rightarrow \mathbb{R}^{+}$, which is continuous w.r.t. its second argument, and such that $L(s, x) \rightarrow 0$ when $|x| \rightarrow \infty$ (where $|x|$ is the Euclidean norm of $x$ in $\mathbb{R}^{d}$ ).

Individual cells We define the cell $C_{0}$ attached to the point $X_{0}$ as the following subset of $\mathbb{R}^{d}$

$$
C_{0}=C_{0}(\Phi)=\left\{y: a_{0} L\left(S_{0}, y-X_{0}\right) \geq b_{0} I_{\Phi}(y)+c_{0}\right\} \text {. }
$$

where $I_{\Phi}(y)$ denotes the value of the shot noise process (see e.g. $[18,14,11,10]$ ) of $\left\{X_{i}, S_{i}\right\}$ at point $y$ for the response function $L$, namely :

$$
I_{\Phi}(y)=\sum_{i} L\left(S_{i}, y-X_{i}\right)=\int_{\mathbb{R}^{d} \times \mathbb{D}} L(s, y-x) \Phi(d(x, s)) .
$$

The second formula is obtained when considering $\Phi$ as the random point measure $\Phi=\sum_{i} \delta_{\left(X_{i}, Z_{i}\right)}$ and when using the simplified notation $\Phi(d(x, s))=\Phi\left(d(x, s) \times(\mathbb{R})^{3}\right)$.

Coverage process The union of all cells

$$
\Xi=\Xi(\Phi)=\bigcup_{i} C_{i}(\Phi)
$$

is the associated coverage process. If $b_{i}>0$ a.s. $\Xi$ can also be expressed as

$$
\Xi=\left\{y: \text { there exists } X_{i} \in \Phi: a_{i} L\left(S_{i}, y-X_{i}\right) \geq b_{i} I_{\Phi}(y)+c_{i}\right\}=\left\{y: M_{\Phi}(y) \geq I_{\Phi}(y)\right\},
$$

where

$$
M_{\Phi}(y)=\max _{i}\left(\frac{a_{i}}{b_{i}} L\left(S_{i}, y-X_{i}\right)-\frac{c_{i}}{b_{i}}\right)
$$

is the so-called max-shot noise process of $\Phi$ (see e.g. [10]) for the response function

$$
L^{\prime}(z, y)=\frac{a}{b} L(s, y)-\frac{c}{b}, \quad z=(s, a, b, c) .
$$

Note that this representation only holds when assuming that the max is well defined, for example when there is an a.s. finite number of cells covering point $y$. 


\subsection{Conditions for the model to be well defined}

We now list some desirable properties which such a model should have to be well defined. We will give some sufficient conditions for these properties to hold under either stationarity or Poisson type assumptions in the following section.

Individual cells Since $L(\cdot)$ is positive, $I_{\Phi}(y)$ is well defined but can be infinite. In the sequel we will require this random function to be a.s. finite and even more, to have finite expectation. Moreover we will require $C_{0}$ (and in fact each cell $C_{i}$ ) to be a random closed set. Since $L$ is a continuous function of its last argument, $C_{0}$ is a.s. a closed set provided $I_{\Phi}(y)$ is also a.s. continuous in $y$ (lower semi-continuity is sufficient).

Coverage process We will also require $\Xi$ to be a random closed set (note that the countable union of closed sets need not be closed). In fact we will require the stronger property that for any given bounded set in $\mathbb{R}^{d}$ (with compact closure) the number of cells that have non-empty intersection with it is almost surely finite. An equivalent statement is that the collection of cells is a.s. a Radon point measure on the space of closed sets, so that it can be treated as a point process

$$
\sum_{i} \delta_{C_{i}(\Phi)}
$$

on the space of closed sets. This is a typical assumption for coverage processes (in particular for the Boolean model, see e.g. [17], eq. (3.1.1), p. 59.).

\subsection{Motivating examples}

Basic example Roughly speaking, a snapshot of a wireless communication network is characterized by the following geometric and technological parameters:

- $X_{i}$ : the location of antenna $i$;

- $S_{i} \in \mathbb{R}^{+}$: the power of the signal emitted from $X_{i}$;

- $\theta_{i} \in \mathbb{R}^{+}$: the signal to noise ratio threshold required for the good reception of the signal emitted by this antenna;

- $W_{i} \in \mathbb{R}^{+}$: the external noise in the vicinity of antenna $i$.

- $l: \mathbb{R}^{3} \rightarrow \mathbb{R}^{+}$, which gives the attenuation function or path-loss of the signal.

Within this setting, we will say that a point $y$ of the plane can receive the signal of station 0 (or equivalently belongs to the cell attached to the antenna located at $X_{0}$ ) if the signal emitted by this station is received at point $y$ at a level $S_{0} l\left(y-X_{0}\right)$ which is at least $\theta_{0}$ times bigger than the total noise, which is here defined as the sum of $W_{0}$ and of all other signals received at $y$, that is

$$
C_{0}=\left\{y: S_{0} l\left(y-X_{0}\right) \geq \theta_{0}\left(I_{\Phi}(y)-S_{0} l\left(y-X_{0}\right)+W_{0}\right)\right\} .
$$

We recognize our generic model with $\mathbb{D}=\mathbb{R}^{+}, L(s, x)=\operatorname{sl}(x), a_{0}=1+\theta_{0}, b_{0}=\theta_{0}$ and $c_{0}=\theta_{0} W_{0}$. 
Signal to interference ratio Consider now the case where the signal emitted by station 0 interferes with the cumulated signals emitted from stations $i \neq 0$ in such a way that only some proportion $\kappa_{0}$ of these cumulated signals should be considered as noise; in the spread spectrum CDMA (code division multiple access) context, this interference stems from the fact that the different codes are not perfectly orthogonal (see e.g. Ehrenberger and Leibnitz [4] or Veeravalli et al [19]). Then one gets another instance of the generic model with

$$
C_{0}=\left\{y: S_{0} l\left(y-X_{0}\right) \geq \theta_{0}\left(\kappa_{0}\left(I_{\Phi}(y)-S_{0} l\left(y-X_{0}\right)\right)+W_{0}\right)\right\} .
$$

Note that this signal to noise ratio principle is not bound to CDMA, and it is actually used as a generic paradigm for wireless communications; see e.g. the physical model in Gupta and Kumar [8].

External noise as a random field In this variant, we replace the external noise sequence $\left\{W_{i}\right\}$ by a shot noise process created by yet another marked point process $\Phi^{\prime}$ with a possibly different attenuation function $l^{\prime}$. This is for instance the case when there are possible interferences between the signals from antennas to mobile phones (the so called down-link, which is considered in the above models) and the signals from the phones to the antennas (the so called up-link).

Under assumptions similar to those of the basic model, this would corresponds to the following cell definition:

$$
C_{0}=\left\{y: S_{0} l\left(y-X_{0}\right) \geq \theta_{0}\left(I_{\Phi}(y)-S_{0} l\left(y-X_{0}\right)+I_{\Phi^{\prime}}(y)\right)\right\}
$$

where

$$
I_{\Phi^{\prime}}(y)=\sum_{i} S_{i}^{\prime} l^{\prime}\left(y-X_{i}^{\prime}\right)
$$

is this second external noise shot noise process; in the above up-link, down-link interference interpretation, the random variables $X_{i}^{\prime}$ represent the locations of mobile phones, the $S_{i}^{\prime}$ their powers and $l^{\prime}$ the corresponding attenuation function.

This is in fact another incarnation of the generic model as the following reduction shows: let $\phi=\left\{x_{i}, z_{i}\right\}$ be the superposition of the two point processes $\Phi$ and $\Phi^{\prime}$. Let $\left\{t_{i}\right\}$ be the sequence defined by $t_{i}=1$ if point $x_{i}$ belongs to $\Phi$ and $t_{i}=0$ otherwise. Let $\mathbb{D}=\mathbb{R}^{+} \times\{0,1\}$. Define

$$
L((s, 1), x)=s l(x), \quad L((s, 0), x)=s l^{\prime}(x)
$$

and let

$$
I_{\Phi}(y)=\sum_{i} L\left(\left(s_{i}, t_{i}\right), y-x_{i}\right)
$$

Then defining

$$
C_{0}=\left\{y: t_{0} L\left(\left(s_{0}, t_{0}\right), y-x_{0}\right) \geq \theta_{0}\left(I_{\phi}(y)-L\left(\left(s_{0}, t_{0}\right), y-x_{0}\right)\right)+\left(1-t_{0}\right)\right\}
$$

we see that the cells of the points of $\Phi^{\prime}$ are empty, whereas those of the points of $\Phi$ are exactly as expected. So this is a special case of the generic model indeed, with $L((s, t), x)$ as above, $a_{0}=t_{0}\left(1+\theta_{0}\right), b_{0}=\theta_{0}$ and $c_{0}=1-t_{0}$ in order to make $C_{0}$ empty when $\left.t_{0}=0\right)$. 
More general attenuation functions Within the setting of the basic model, the attenuation function is often taken of the form $l(x)=|x|^{-\alpha},(1+|x|)^{-\alpha}$ or $\left.(\max (R,|x|))^{-\alpha}\right)$, which corresponds to the case of isotropic antennas with ideal Hertzian propagation. When antennas are directional, a mark $T_{i} \in \mathbb{R}^{d}$ should be used for describing the direction of antenna $i$ in the $d$ dimensional Euclidean space; the effect of antenna $i$ on point $y$ can then for instance be defined by a function of the type

$$
L\left(\left(S_{i}, T_{i}\right), y-X_{i}\right)=S_{i} h\left(T_{i}, y-X_{i}\right)
$$

Even in the isotropic antenna case, in most practical situations, propagation is altered by fading and/or reflections. For representing this, it is possible to use mark $T_{i}$ to describe the random component of the loss on the path from antenna $i$ to point $y$.

Randomness sources Besides external noise, randomness is present in such models at several levels: the location of antennas may be a random process like for instance in ad hoc networks, where antennas are mobile. Even in the fixed antenna case, the locations are usually non regular, and can then be seen as a realization of a random point process (see e.g. [1]). Similarly, the powers and ratios are determined from complex adaptive schemes, and it makes sense to adopt a random representation for a temporal snapshot of these variables.

\subsection{Special cases}

Some special examples can be retrieved from our general model when assuming particular values for $a, b, c$. Here are two examples.

Independent cells Suppose $b=0$ a.s. Then the shape of the cell attached to a point $X_{i}$ depends only on $X_{i}$ and its mark $Z_{i}$. If in addition the underlying process $\Phi$ is a Poisson p.p., then $\Xi$ is the well known Boolean model. We will say more on this in Section 6.1.

Level sets of the shot noise If $a=0$ a.s and $b c<0$ then all cells are level sets of the shot-noise process $I_{\Phi}(\cdot)$.

\subsection{Probabilistic assumptions}

Poisson case Several computational results of the paper will be derived under the following assumptions: $\Phi$ is an independently marked Poisson point process (Poisson p.p. for simplicity) where the marks $\left\{Z_{i}\right\}$ constitute a sequence of independent identically distributed random vectors characterized by the distribution of $Z_{0}$. We sometimes use $Z$ for a generic random mark. The default option is that when the underlying (non marked) Poisson process is non homogeneous; we will denote $\mu(\cdot)$ its intensity measure and assume that $\mu$ is non-atomic; thus $\Phi$ is a simple p.p.

Stationary ergodic case Some existence results can be proved using Palm calculus. For this general setting, we will mainly consider the case when the marked point process $\Phi$ is stationary and ergodic, with (constant) intensity $\lambda$.

In what follows, in order to avoid degeneracy and/or special cases, we make the following general assumptions: $a, b, c \in \mathbb{R}^{+}$a.s. and $\mathrm{P}\left(a_{0}=c_{0}=0\right)=0$. 


\section{Sufficient conditions for the model to be well defined}

\subsection{Individual cells}

A well-know necessary and sufficient condition for $I_{\Phi}(y)$ to have finite expectation is

$$
\mathrm{E}\left[I_{\Phi}(y)\right]=\int_{\mathbb{R}^{d} \times \mathbb{D}} L(s, y-x) \mu(d x) H(d s)<\infty,
$$

where $H$ denotes the law of $S_{0} \in \mathbb{D}$ (see e.g. [15]).

As indicated above, a sufficient condition for each individual cell to be a random closed set is the a.s. path-wise continuity of the shot noise process. Here is a simple sufficient condition for this to hold.

Proposition 3.1 Under the Poisson p.p. assumptions, if for each $y \in \mathbb{R}^{d}$, there exists a ball $B\left(y, \epsilon_{y}\right)$ such that

$$
\int_{\mathbb{R}^{d} \times \mathbb{D}} \sup _{z \in B\left(y, \epsilon_{y}\right)} L(s, z-x) \mu(d x) H(d s)<\infty,
$$

then with probability 1 , the function $I_{\Phi}(y)$ is continuous w.r.t. $y$.

Proof: From (3.6) we obtain that there exists a subset $\Omega^{\prime}$ of probability one (of the space on which $\Phi$ is defined) such that for all $\omega \in \Omega^{\prime}$

$$
\int_{\mathbb{R}^{d} \times \mathbb{D}} \sup _{z \in B\left(y, \epsilon_{y}\right)} L(s, z-x) \Phi(\omega)(d(x, s))<\infty
$$

for all $y$ in a countable and dense subset of $\mathbb{R}^{d}$ (for instance the set of rational numbers). We prove that for each $\omega \in \Omega^{\prime}$ the function $I_{\Phi(\omega)}(y)$ is continuous. Take any $y \in \mathbb{R}^{d}$ and $y_{n} \rightarrow y$. For sufficiently large $n, y_{n}$ and $y$ belong to $B\left(w, \epsilon_{w}\right)$ for some rational $w$. Then

$$
\begin{aligned}
I_{\Phi}\left(y_{n}\right) & =\int_{\mathbb{R}^{d} \times \mathbb{D}} L\left(s, y_{n}-x\right) \Phi(d(x, s)) \\
& \leq \int_{\mathbb{R}^{d} \times \mathbb{D}} \sup _{z \in B\left(w, \epsilon_{w}\right)} L(s, z-x) \Phi(d(x, s))<\infty
\end{aligned}
$$

and by the dominated convergence theorem

$$
\lim _{n \rightarrow \infty} I_{\Phi}\left(y_{n}\right)=\int_{\mathbb{R}^{d} \times \mathbb{D}} \lim _{n \rightarrow \infty} L\left(s, y_{n}-x\right) \Phi(d(x, s))=I_{\Phi}(y)
$$

because $L$ is continuous in its second argument.

Stationary ergodic case Analogous results to (3.5) and Proposition 3.1 hold true, with $\mu(d x) H(d s)$ replaced by $\lambda d x H(d s)$, with $H$ being the Palm distribution of the mark $S$. Proofs are based on Campbell's formula (see e.g. [17], eq. (4.4.3), p. 119). 


\subsection{The coverage process}

Denote by $N_{K}$ the random number of cells $C_{i}$ that hit a given bounded set $K$

$$
N_{K}=\sum_{i} \mathbb{I}\left(K \cap C_{i} \neq \emptyset\right)
$$

In the following theorem we give several instances of moment-conditions (bearing on the distribution of $Z_{0}$ and the intensity measure $\mu$ ) for $\mathrm{E}\left[N_{K}\right]$ to be finite for arbitrary large $K$. Note first, that this property is always satisfied if $\mu\left(\mathbb{R}^{d}\right)<\infty$. In the following we will assume one of the two following conditions on the response function:

(A1) There exists a finite real number $R^{*}$, such that $L(s, z)=0$ for all $s \in \mathbb{D}$ and $z \in \mathbb{R}^{d}$ with $|z|>R^{*}$.

(A2) There exist positive constants $\alpha$ and $\beta$ such that $L(s, z) \leq \beta\|s\||z|^{-\alpha}$, for all (s,z), where $\|s\|$ denotes the norm of $s \in \mathbb{D}$.

\section{Proposition 3.2 We have}

$$
\mathrm{E}\left[N_{K}\right]<\infty
$$

for an arbitrary large $K$ if one of the following conditions holds:

(i) Condition (A1) is satisfied and a.s. $c_{0}>0$,

(ii) Condition (A2) is satisfied, $c_{0}>0$ a.s., and for all $R>0$

$$
\mathrm{E}\left[\mu\left(B\left(0, R+\left(\frac{\beta a_{0}\left\|S_{0}\right\|}{c_{0}}\right)^{1 / \alpha}\right)\right)\right]<\infty .
$$

(iii) Condition (A2) is satisfied, $b_{0}>0$ a.s, $L(S, y)>0$ a.s. for all $y \in \mathbb{R}^{d}$, and for all $R>0$

$$
\int_{\mathbb{R}^{d}} e^{-\mu(B(0,|x|))} \mathrm{E}\left[\mu\left(B\left(0, R+\left(\frac{\beta a_{1}\left\|S_{1}\right\|}{b_{1} \underline{L}\left(S_{0},|x|+R\right)}\right)^{1 / \alpha}\right)\right)\right] \mu(d x)<\infty,
$$

where $S_{0}$ is independent of $\left(S_{1}, a_{1}, b_{1}\right)$, with both having the distribution of the marginals of a typical mark, and $\underline{L}(s, r)=\inf _{z,|z| \leq r} L(s, z)$.

Proof: In order to prove (3.8), we will construct various Boolean models dominating our coverage process $\Xi$ and we will use the following standard result (see e.g. Stoyan et al [17] p. 65) : for a Boolean with intensity measure $\mu$ and generic random cell $G$

$$
\mathrm{E}\left[\sum_{X_{i} \in \Phi} \mathbb{I}\left(K \cap\left(G_{i} \oplus X_{i}\right) \neq \emptyset\right)\right]=\mathrm{E}[\mu(K \oplus \check{G})]
$$

In particular, finiteness of the right hand side is necessary and sufficient to ensure that the number of cells of the Boolean model which intersect $K$ is of finite mean.

(i) Under (A1), we have $C_{i} \subset B\left(X_{i}, R^{*}\right)$ and the result follows from the fact that (3.11) is obviously finite for the Boolean model with deterministic cells. 
(ii) Under (A2) we have

$$
\begin{aligned}
C_{i} & =\left\{y: a_{i} L\left(S_{i}, y-X_{i}\right) \geq b_{i} I_{\Phi}(y)+c_{i}\right\} \\
& \subset\left\{y: a_{i} L\left(S_{i}, y-X_{i}\right) \geq c_{i}\right\} \\
& \subset\left\{y: \beta a_{i}\left\|S_{i}\right\|\left|y-X_{i}\right|^{-\alpha} \geq c_{i}\right\} \\
& \subset\left\{y:\left|y-X_{i}\right| \leq\left(\frac{\beta a_{i}\left\|S_{i}\right\|}{c_{i}}\right)^{1 / \alpha}\right\} .
\end{aligned}
$$

Thus we have $C_{i} \subset B\left(X_{i}, \rho_{i}\right)$, a.s., where

$$
\rho_{i}=\left(\frac{\beta a_{i}\left\|S_{i}\right\|}{c_{i}}\right)^{1 / \alpha}
$$

There is no loss of generality in assuming that the bounded set $K$ is the ball $B(0, R)$ and the result now follows from the simple observation that $B(0, R) \oplus B\left(0, \rho_{i}\right)=B\left(0, R+\rho_{i}\right)$.

(iii) Now we do not assume anything about $c_{0}$ (thus it may by positive or null). Instead we will use one of the points of the process $\Phi$ to guarantee a sufficient level for the variable $I_{\Phi}$ and thus bound cell sizes from above. Let $X_{0}$ denote the point of $\Phi$ which is the nearest to the origin, and let $Z_{0}$ be its mark. We have

$$
\begin{aligned}
N_{K} & =\mathbb{I}\left(K \cap C_{0} \neq \emptyset\right)+\sum_{i \neq 0} \mathbb{I}\left(K \cap C_{i} \neq \emptyset\right) \\
& \leq 1+\sum_{i \neq 0} \mathbb{I}\left(K \cap C_{i} \neq \emptyset\right) .
\end{aligned}
$$

For any point $X_{i} \neq X_{0}$ (i.e., $\left|X_{i}\right|>\left|X_{0}\right|$ ) of the point process, with mark $Z_{i}$,

$$
\begin{aligned}
C_{i}(\Phi) & \subset\left\{y: a_{i} L\left(S_{i}, y-X_{i}\right) \geq b_{i} L\left(S_{0}, y-X_{0}\right)+c_{i}\right\} \\
& \subset\left\{y: \beta a_{i}\left\|S_{i}\right\|\left|y-X_{i}\right|^{-\alpha} \geq b_{i} L\left(S_{0}, y-X_{0}\right)\right\} \\
& \subset\left\{y:\left|y-X_{i}\right| \leq\left(\frac{\beta a_{i}\left\|S_{i}\right\|}{b_{i} L\left(S_{0}, y-X_{0}\right)}\right)^{1 / \alpha}\right\} .
\end{aligned}
$$

Moreover, for $K=B(0, R)$

$$
\begin{aligned}
C_{i}(\Phi) \cap K & \subset\left\{y:|y| \leq R \text { and }\left|y-X_{i}\right| \leq\left(\frac{\beta a_{i}\left\|S_{i}\right\|}{b_{i} L\left(S_{0}, y-X_{0}\right)}\right)^{1 / \alpha}\right\} \\
& \subset\left\{y:\left|y-X_{i}\right| \leq\left(\frac{\beta a_{i}\left\|S_{i}\right\|}{b_{i} \inf _{y,|y| \leq R} L\left(S_{0}, y-X_{0}\right)}\right)^{1 / \alpha}\right\} \\
& \subset B\left(X_{i}, \rho\left(R, Z_{i}, X_{0}, S_{0}\right)\right),
\end{aligned}
$$

$\mathrm{RR} \mathrm{n}^{\circ} 4019$ 
where

$$
\rho\left(R, Z_{i}, X_{0}, S_{0}\right)=\left(\frac{\beta a_{i}\left\|S_{i}\right\|}{b_{i} \underline{L}\left(S_{0}, R+\left|X_{0}\right|\right)}\right)^{1 / \alpha} .
$$

Using now (3.14) and the assumption that $X_{0}$ is the point nearest to the origin, we get

$$
\begin{aligned}
\mathrm{E}\left[N_{K}\right] & \leq \mathrm{E}\left[1+\sum_{i \neq 0} \mathbb{I}\left(K \cap C_{i} \neq \emptyset\right)\right] \\
& =\mathrm{E}\left[\int_{\mathbb{R}^{d}} \mathbb{I}\left(\Phi\left(B^{o}\left(0, x_{0}\right)\right)=0\right)\left(1+\sum_{i \neq 0} \mathbb{I}\left(K \cap C_{i} \neq \emptyset\right)\right) \Phi\left(d x_{0}\right)\right] \\
& \leq \int_{\mathbb{R}^{d}} e^{-\mu\left(B\left(0,\left|x_{0}\right|\right)\right)} \mathrm{E}\left[1+\sum_{i,\left|X_{i}\right|>x_{0}} \mathbb{I}\left(K \cap B\left(X_{i}, \rho\left(R, Z_{i}, x_{0}, S_{0}\right)\right) \neq \emptyset\right)\right] \mu\left(d x_{0}\right) .
\end{aligned}
$$

So by (3.11) for the Boolean model with $G_{i}=B\left(0, \rho\left(R, Z_{i}, x_{0}, S_{0}\right)\right)$ conditioned on $S_{0}$

$$
\mathrm{E}\left[N_{K}\right] \leq \int_{\mathbb{R}^{d}} e^{-\mu\left(B\left(0,\left|x_{0}\right|\right)\right)} \mathrm{E}\left[1+\mu\left(K \oplus B\left(0, \rho\left(R, Z_{1}, X_{0}, S_{0}\right)\right)\right)\right] \mu(d x)
$$

The proof is concluded by observing that $B(0, R) \oplus B(0, \rho(\ldots))=B(0, R+\rho(\ldots))$.

Corollary 3.3 Let $\Phi$ be an independently marked and homogeneous Poisson p.p. with intensity $\mu(d x)=\lambda d x$. Then (3.9) is equivalent to the following condition

$$
\mathrm{E}\left[\left(\frac{a_{0}\left\|S_{0}\right\|}{c_{0}}\right)^{d / \alpha}\right]<\infty
$$

whereas (3.10) is equivalent to the conjunction of the following two conditions

$$
\begin{aligned}
\int_{\mathbb{R}^{d}} e^{-\lambda \mathbf{b}_{d}|x|^{d}}\left(\mathrm{E}\left[\underline{L}\left(S_{0},|x|+R\right)\right]\right)^{-d / \alpha} d x & <\infty \\
\mathrm{E}\left[\left(\frac{a_{0}\left\|S_{0}\right\|}{b_{0}}\right)^{d / \alpha}\right] & <\infty .
\end{aligned}
$$

Stationary ergodic case Conditions analogous to part (i) and (ii) of Proposition 3.2 can be observed in the stationary ergodic case; (3.9) and (3.15) have the same form with E[...] replaced with $\mathrm{E}_{0}[\ldots]$, where $\mathrm{E}_{0}$ is the expectation w.r.t. the Palm distribution of the mark $\left(S_{0}, a_{0}, c_{0}\right)$. The proof is based on Campbell's formula. Part (iii) has no generalization due to the lack of an explicit form of the joint distribution of $X_{0}$ (the point which is nearest to the origin) and the remaining part of a general point process. 


\section{Typical cell characteristics}

In this section we want to analyze the cell $C(x ; \Phi)$ attached to a point located at $x$ of the marked Poisson p.p. $\Phi$ under the Palm distribution $\mathrm{P}_{x}$. Due to Slivnyak's theorem, the law of this set under $\mathrm{P}_{x}$ is the same as that of the random closed set

$$
C\left(x ; \Phi+\delta_{(x, Z)}\right)
$$

under $\mathrm{P}$, where $\Phi$ is the original Poisson p.p. and $Z=(S, A)=(S,(a, b, c))$ is an "additional mark" distributed like the other marks and independent of $\Phi$. We will refer to (4.1) as the typical cell located at $x$. If the Poisson point process is homogeneous, the characteristics are the same for all points $x$ and we will speak of the typical cell.

Coverage probability Denote by $p_{x}(y)$ the probability that point $y \in \mathbb{R}^{d}$ is covered by $C\left(x ; \Phi+\delta_{(x, Z)}\right)$. We have

$$
\begin{aligned}
p_{x}(y)= & \mathrm{P}\left(y \in C\left(x ; \Phi+\delta_{(x, Z)}\right)\right) \\
= & \mathrm{P}\left((a-b) L(S, y-x)-b I_{\Phi}(y)-c \geq 0\right) \\
= & \mathrm{P}((a-b) L(S, y-x)-c \geq 0, b=0) \\
& +\mathrm{P}\left(\left(\frac{a}{b}-1\right) L(S, y-x)-\frac{c}{b}-I_{\Phi}(y) \geq 0 \mid b>0\right) \mathrm{P}(b>0) .
\end{aligned}
$$

The distribution of the mark $Z$ can be considered as given. Note also that the random variables $I_{\Phi}(y)$ and $Z$ involved in (4.2) are independent. Thus in order to determine the probability $p_{x}(y)$, we need to know the marginal distribution of the shot-noise process $I_{\Phi}(\cdot)$ at $y$. This distribution is usually not known explicitly, but only via its transforms. The characteristic functional of the process $I_{\Phi}(\cdot)$ is given by

$$
\begin{aligned}
\varphi_{I}(\nu) & =\operatorname{Eexp}\left[i \int_{\mathbb{R}^{d}} I_{\Phi}(y) \nu(d y)\right] \\
& =\exp \left[\int_{\mathbb{R}^{d} \times \mathbb{D}}\left(\exp \left[i \int_{\mathbb{R}^{d}} L(s, y-x) \nu(d y)\right]-1\right) \mu(d x) H(d s),\right.
\end{aligned}
$$

where $\nu$ is any measure on $\mathbb{R}^{d}$ such that the outer integral in (4.3) is finite (see [14] for example). The joint characteristic function of the vector $\left(I_{\Phi}\left(y_{1}\right), \ldots, I_{\Phi}\left(y_{n}\right)\right)$ can be obtained from (4.3) by setting $\nu=\sum_{k=1}^{n} \xi_{k} \delta_{y_{k}}$.

The distribution function of $I_{\phi}(y)$ is usually not known in closed form, but for special cases (e.g. for exponential response function and exponential marks, it is gamma-distributed; cf. [13]). For results on the approximation of its distribution function and density, we refer to [7] and the literature cited therein.

Note however that in order to obtain $p_{x}(y)$, we do not need the whole distribution function of $I_{\Phi}(y)$. Knowing the transforms of $I_{\Phi}(y)$ and of $(a / b-1) L(S, y-x)-c / b$ (conditionally on $\left.b>0\right)$, we only have to compute the probability for a real valued random variable to be nonnegative from the knowledge of its Fourier transform. In the Appendix we show how to reduce this to the solution of a Riemann boundary problem on the real line. Under the additional assumption that the random variable in question has a density (with respect to Lebesgue measure) The 
solution of this problem can be expressed as a singular integral transformation of the Fourier transform known as Hilbert's transform. Since in our case this random variable is the difference $(a / b-1) L(S, y-x)-c / b-I_{\Phi}(y)$ it suffices that one of the terms has a density for the whole sum to have one. Some conditions for this to hold for $I_{\Phi}(y)$ are given in the Appendix, together with comments on the numerical evaluation of this singular integral.

Note that the mean value of the Lebesgue measure $\nu_{d}\left(C\left(x ; \Phi+\delta_{(x, Z)}\right)\right)$ of the typical cell located at point $x$, is then obtained from the $p_{x}(\cdot)$ function by the relation:

$$
\mathrm{E}\left[\nu_{d}\left(C\left(x ; \Phi+\delta_{(x, Z)}\right)\right)\right]=\int_{\mathbb{R}^{d}} p_{x}(y) d y
$$

Example 4.1 Consider a homogeneous Poisson p.p. $\Phi$ in the plane (i.e. $d=2$ and $\mu(x) \equiv$ $1)$. We assume $L(s, z)=s\left(\max (|z|, R)^{-4}\right.$. The random variable $S \in \mathbb{R}^{+}$is assumed to be exponential with mean $m$. Then, direct computations show that the Fourier transform of $I_{\Phi}=I_{\Phi}(y)$ does not depend on $y$ and is equal to

$$
\psi_{I_{\Phi}}(\xi i)=\mathrm{E}\left[e^{-i \xi I_{\Phi}}\right]=\exp \left[\pi \sqrt{\frac{i \xi}{m}} \arctan \left(R^{2} \sqrt{\frac{m}{i \xi}}\right)-\frac{1}{2} \pi^{2} \sqrt{\frac{i \xi}{m}}\right],
$$

for $\xi \in \mathbb{R}$, where the branch of the complex square root function is chosen with positive real part. Moreover, we take $R=1,|y-x|=1, m=1$ and $c=1, b=\epsilon, a=1+\epsilon$ a.s. Using the Fourier transform of $L(S, y-x)-1-\epsilon I_{\Phi}(y)$ and the integral formula (A.1) in the Appendix, we calculated numerically $p_{x}(y)$ using the method described in [6] for computing singular integrals. Obviously for $\epsilon=0$ there is no shot-noise part in our model, and $p_{x}(y)$ can be easily calculated without any contour integration. Some results are presented in Table 1 (see also Figure 3 (a) in Section 6.1.2).

\begin{tabular}{|c||c|c|c|c|c|c|c|c|c|c|c|c|c|}
\hline$\epsilon$ & 1 & .9 & .8 & .7 & .6 & .5 & .4 & .3 & .2 & .1 & .05 & .02 & 0 \\
\hline$p_{x}(y)$ & .006 & .009 & .012 & .016 & .023 & .033 & .049 & .075 & .121 & .204 & .271 & .329 & .368 \\
\hline
\end{tabular}

Table 1: Probabilities $p_{x}(y)$ numerically calculated using Formula (A.1).

Covariance The probability $p_{x}\left(y_{1}, y_{2}\right)$ that two given points $y_{1}, y_{2} \in \mathbb{R}^{d}$ are covered by the typical cell located at $x$ (sometimes called the covariance of the typical cell) can be expressed

$$
\begin{aligned}
p_{x}\left(y_{1}, y_{2}\right) & =\mathrm{P}\left(y_{i} \in C\left(x ; \Phi+\delta_{(x, Z)}\right), i=1,2\right) \\
& =\mathrm{P}\left((a-b) L\left(S, y_{i}-x\right)-b I_{\Phi}\left(y_{i}\right)-c \geq 0, \quad i=1,2\right) .
\end{aligned}
$$

Again, the pair $\left(I_{\Phi}\left(y_{1}\right), I_{\Phi}\left(y_{2}\right)\right)$ is independent of other variables involved in the formula, and the the joint characteristic function of the vector $\left(I_{\Phi}\left(y_{1}\right), I_{\Phi}\left(y_{2}\right)\right)$ can be derived form (4.3).

\section{$5 \quad$ The coverage process characteristics}

Our goal in this section is to analyze the coverage process $\Xi(\Phi)$, and more specifically, the distribution of the number of cells covering a given point. From this, the volume fraction and 
other characteristics of $\Xi(\Phi)$ can be derived. Let $N_{x}=N_{\{x\}}$ (cf. (3.7)) denote the number of cells covering a given point $x$. For all integers $k$, let $k^{(n)}=k(k-1) \ldots(k-n+1)^{+}$, where $k^{+}=\max (0, k)$. Below we give formulas for factorial moments $\mathrm{E}\left[N_{x}^{(n)}\right]$ of $N_{x}$. From this, the distribution of $N_{x}$ can be derived using the formula

$$
\mathrm{P}\left(N_{x}=n\right)=\frac{1}{n !} \sum_{k=0}^{\infty}(-1)^{k} \frac{\mathrm{E}\left[N_{x}^{(n+k)}\right]}{k !},
$$

which follows from the well-known expansion of the generating function. Of course, these expansions usually require strong conditions (existence of all moments and convergence of the series). We will later give some sufficient conditions for this to hold. But before we proceed along these lines, we make a purely algebraic observation concerning the possibility of intersection of several cells.

\subsection{Intersection of cells}

Suppose the following $n$ sets are given

$$
C_{i}=\left\{y \in \mathbb{R}^{d}: a_{i} L\left(s_{i}, y-x_{i}\right) \geq b_{i} \sum_{j=1}^{n} L\left(s_{j}, y-x_{j}\right)+c_{i}\right\}, \quad i=1, \ldots, n,
$$

where $\left(x_{i},\left(S_{i},\left(a_{i}, b_{i}, c_{i}\right)\right) \in \mathbb{R}^{d} \times\left(\mathbb{D} \times\left(\mathbb{R}^{+}\right)^{3}\right)\right.$ and $a_{i}, c_{i}>0, i=1, \ldots, n$, are given vectors.

Lemma 5.1 The following inequality

$$
\sum_{i=1}^{n} \frac{b_{i}}{a_{i}}<1
$$

is a necessary condition for the set of cells $C_{i}, i=1, \ldots, n$, given by (5.6) to have a common nonempty intersection. If it holds then

$\bigcap_{i=1}^{n} C_{i}=\left\{y: L\left(s_{i}, y-x_{i}\right)=\frac{c_{i} / a_{i}+b_{i} / a_{i} \sum_{j=1}^{n} \zeta_{j}}{1-\sum_{j=1}^{n} b_{j} / a_{j}}+\zeta_{i}(i=1, \ldots, n)\right.$ for some $\left.\zeta_{1}, \ldots, \zeta_{n} \geq 0\right\}$.

Remark: Note that Lemma 5.1 says that the cells (5.6) must have empty intersection if $\sum_{t=1}^{n} b_{i} / a_{i} \geq 1$, and this regardless of the proximity of the points $x_{i}(i=1, \ldots, n)$ and of the values of the $s_{i}$. In the CDMA literature, this kind of condition is related to the existence of the so called pole capacity (see e.g. [19]). Note also that the condition (5.7) is not an iff condition; i.e., it can be satisfied and the intersection (5.8) can still be empty. But it is sufficient in the following sense: if it is satisfied and $L\left(s, y-x_{i}\right) \rightarrow \infty$ when $\|s\| \rightarrow \infty$ for some $y$ and all $i=1, \ldots n$, then there exist $s_{1}, \ldots, s_{n}$ (with possibly very large norm) such that the intersection is not empty (it contains $y$ ).

Proof of Lemma 5.1 The set of inequalities defining the intersection of the cells (5.6)

$$
L\left(s_{i}, y-x_{i}\right) \geq \frac{b_{i}}{a_{i}} \sum_{j=1}^{n} L\left(s_{j}, y-x_{j}\right)+\frac{c_{i}}{a_{i}}
$$

$\mathrm{RR} \mathrm{n}^{\circ} 4019$ 
implies (by adding them up and rearranging)

$$
\left(1-\sum_{j=1}^{n} \frac{b_{i}}{a_{i}}\right) \sum_{i=1}^{n} L\left(s_{i}, y-x_{i}\right) \geq \sum_{j=1}^{n} \frac{c_{i}}{a_{i}}
$$

and for $c_{i}>0$, the right hand side is strictly bigger than 0, thus the necessity of Condition (5.7) follows. Assume now it is satisfied. The set of inequalities (5.9) can be rewritten equivalently under the matrix form

$$
\mathrm{B} \sigma \geq \kappa
$$

where $\mathbf{B}=\mathbf{I}-\boldsymbol{\tau} \mathbf{1}^{t}, \boldsymbol{\sigma}, \boldsymbol{\tau}, \boldsymbol{\kappa}$ are the column vectors with coordinates $L\left(s_{i}, y-x_{i}\right), b_{i} / a_{i}$ and $c_{i} / a_{i}(i=1, \ldots, n)$, respectively, $\mathbf{I}$ is the $n$-dimensional identity matrix and $\mathbf{1}^{t}=(1,1, \ldots, 1)$. The matrix $\mathbf{B}$ is nonsingular, and its inverse is equal to $\mathbf{B}^{-1}=\mathbf{I}+\boldsymbol{\tau} \mathbf{1}^{t} /\left(1-\mathbf{1}^{t} \boldsymbol{\tau}\right)$. So $\boldsymbol{\sigma}$ satisfies the inequality (5.11) whenever it is of the form

$$
\boldsymbol{\sigma}=\mathbf{B}^{-1}\left(\boldsymbol{\kappa}+\operatorname{diag}\left(\zeta_{1}, \ldots, \zeta_{n}\right) \mathbf{1}\right) \text { for some } \zeta_{1}, \ldots, \zeta_{n} \geq 0,
$$

where $\operatorname{diag}(\ldots)$ is the diagonal matrix with $(\ldots)$ on its diagonal. Calculating the entries of $\mathbf{B}^{-1}\left(\boldsymbol{\kappa}+\operatorname{diag}\left(\zeta_{1}, \ldots, \zeta_{n}\right) \mathbf{1}\right)$, we get $(5.8)$.

Suppose now that the distribution of the mark $A_{0}=\left(a_{0}, b_{0}, c_{0}\right)$ is such that $b_{0} / a_{0}$ is bounded away from 0; i.e.,

(B) $b_{0} / a_{0} \geq \rho$ a.s. for some constant $\rho>0$,

Using the result of Lemma 5.1 we immediately have the following property of the coverage process.

Corollary 5.2 If Condition (B) is satisfied and one of the following conditions holds: (i) $c>0$ a.s. or (ii) $\mu\left(\mathbb{R}^{d}\right)=\infty$ and $L(S, x)>0$ a.s. for all $x \in \mathbb{R}^{d}$, then $N_{x}<1 / \rho$ almost surely.

Proof: Assume that $n=N_{x}$ cells cover point $x$. Let $\Phi^{\prime}$ be the realization of the Poisson p.p. $\Phi$ from which one subtracts the $n$ points generating the cells covering $x$. Let $c_{i}^{\prime}=c_{i}+I_{\Phi^{\prime}}(x)$, where $c_{i}$ is the mark of point $X_{i}$. Note that if $c_{i}>0$ a.s. or if $\mu\left(\mathbb{R}^{d}\right)=\infty$ and $L(S, \cdot)>0$, then $c_{i}^{\prime}>0$ a.s. Note also that the $a_{i}$ marks of these $n$ points are necessarily positive (otherwise the intersection would be empty). From condition (B),

$$
\sum_{1}^{N_{x}} \frac{b_{i}}{a_{i}} \geq N_{x} \rho
$$

Since by assumption, the $n$ cells have a nonempty intersection, it follows from Lemma 5.1 applied to these $n$ points with the parameters $a_{i}, b_{i}$ and $c_{i}^{\prime}$ that $N_{x} \rho<1$.

Remark: This bound suggests an analogy with queueing theory. One can think of queueing theory as a way of sharing time between customers arriving at a queue according to some point process on the line, and requiring some given service times. We can also think of our coverage process as a way of sharing space between the points of a spatial point process with given marks. Under the condition mentioned in the last lemma, the coverage process can be seen as a spatial analogue of the $\ell$-server queue, with $\ell=\min \{n$ integer $: n \geq 1 / \rho\}$, in that no 
point in space can be covered by more than $\ell$ cells; in the same way, the $\ell$-server queue forbids that at any point in time, more than $\ell$-customers could be served. Note that sharing actually means quite different things here and there: in queues, the sharing of time is implemented by shifting customers in excess to later times, while keeping their service times unchanged. In contrast, for this coverage process, sharing of space is obtained by shrinking the marks: if one defines the space request of point $x_{0}$ as the set $C_{0}^{(0)}=\left\{y: a_{0} L\left(s_{0}, y-x_{0}\right) \geq c_{0}\right\}$, which would be the share of space obtained by $x_{0}$ if there were no other points, then one can see the set $C_{0}=\left\{y: a_{0} L\left(s_{0}, y-x_{0}\right) \geq b_{0} I_{\Phi}(y)+c_{0}\right\}$, as a shrunken version of $C_{0}^{(0)}$ resulting from the competition with the other points.

In the same vein, we will show later on that the Boolean model, which is a limiting case of our coverage process, can also be seen as a spatial analogue of the infinite server queue, and that in this case, the analogy is quite strong, with in particular the same Poisson distribution for the number of marks (customers or cells) covering a given (time or space) point.

\subsection{Factorial moments of $N_{x}$}

We are now in a position to prove the following result.

Proposition 5.3 The $n$-th factorial moment of the number $N_{x}$ of cells of $\Xi(\Phi)$ covering point $x$ is equal to

$$
\mathrm{E}\left[N_{x}^{(n)}\right]=\int_{\left(\mathbb{R}^{d}\right)^{n}} \mathrm{P}\left(x \in \bigcap_{k=1}^{n} C\left(x_{k} ; \Phi+\sum_{i=1}^{n} \delta_{\left(x_{i}, Z_{i}\right)}\right)\right) \mu\left(d x_{1}\right) \ldots \mu\left(d x_{n}\right),
$$

where $\Phi$ is the Poisson p.p. and $\left\{Z_{i}\right\}_{i=1}^{n}$ is an independent sequence of mutually independent vectors distributed as the generic mark; this relation holds provided the integral on the right hand side is finite.

Corollary 5.4 If $\Phi$ is a homogeneous Poisson p.p. with intensity $\mu(d x)=\lambda d x$ then for each $x \in \mathbb{R}^{d}$

$$
\mathrm{E}\left[N_{x}^{(n)}\right]=\mathrm{E}\left[N_{0}^{(n)}\right]=\lambda^{n} \int_{\left(\mathbb{R}^{d}\right)^{n}} \mathrm{P}\left(0 \in \bigcap_{k=1}^{n} C\left(x_{k} ; \Phi+\sum_{i=1}^{n} \delta_{\left(x_{i}, Z\right)}\right)\right) d x_{1} \ldots d x_{n}
$$

provided the integral is finite. In particular, for $n=1$

$$
\mathrm{E}\left[N_{0}\right]=\lambda \mathrm{E}\left[\nu_{d}\left(C\left(0 ; \Phi+\delta_{(0, Z)}\right)\right)\right]
$$

where $\nu_{d}(C(\ldots))$ is the d-dimensional volume of the typical cell.

Proof of Proposition 5.3: for a particular realization $\Phi=\left\{X_{i}, Z_{i}\right\}_{i}$ of the marked Poisson p.p. $\Phi$, denote by $\Phi^{(n)}$ its $n$-th factorial power, that is the following point measure on $\left(\mathbb{R}^{d} \times \mathbb{D} \times\left(\mathbb{R}^{+}\right)^{3}\right)^{n}$

$$
\Phi^{(n)}=\left\{\left(\left(X_{i_{1}}, \ldots, X_{i_{n}}\right),\left(Z_{i_{1}}, \ldots, Z_{i_{n}}\right)\right)\right\}_{\substack{X_{i_{1}}, \ldots, X_{i_{n}} \in \Phi \\ \text { distinct }}}
$$


In other words, $\Phi^{(n)}$ consists of all $n$-tuples of distinct points of $\Phi$. Now we can write the factorial power $\left(N_{x}\right)^{(n)}$ of the number cells covering point $x$ as the following integral with respect to $\Phi^{(n)}$ using the simplified notation $\Phi^{(n)}\left(d\left(x_{1}, \ldots, x_{n}\right)\right)=\Phi^{(n)}\left(d\left(x_{1}, \ldots, x_{n}\right) \times\left(\mathbb{D} \times\left(\mathbb{R}^{+}\right)^{3}\right)^{n}\right)$

$$
N_{x}^{(n)}=\int_{\left(\mathbb{R}^{d}\right)^{n}} \prod_{k=1}^{n} \mathbb{I}\left(x \in C\left(x_{k} ; \Phi\right)\right) \Phi^{(n)}\left(d\left(x_{1}, \ldots, x_{n}\right)\right) .
$$

We get (5.12) by applying the refined Campbell theorem to the expectation of this integral and the Slivnyak's theorem.

Remark: For the finiteness of the integral that appears in Proposition 5.3 (or Corollary 5.4) in the case $c>0$ a.s. it is enough to assume exactly the same conditions as for the $\sigma$-finiteness of the mean measure of $\sum_{i} \delta_{C_{i}}$ given in Proposition 3.2 part (i) and (ii). In the case $\mathrm{P}(c=0)>0$ however, some integrals of the negative moments of order $n d / \alpha$ of $L\left(S, x-X_{0}\right)$ are to be finite, where $X_{0}$ is the point which is nearest to the origin. We show this in the following paragraph.

Moment conditions for $N_{x}$ Note first that if $\mu\left(\mathbb{R}^{d}\right)<\infty$ then all moments of $N_{x}$ are finite.

Proposition 5.5 The integral (5.12) is finite if one of the following conditions holds:

(i) Condition (A1) is satisfied and $c_{0}>0$ a.s.,

(ii) Condition (A2) is satisfied, $\mathrm{P}\left(c_{0}=b_{0} I_{\Phi}(x)=0\right)=0$ and

$$
\mathrm{E}\left[\prod_{k=1}^{n} \mu\left(B\left(x,\left(\beta \frac{a_{k}\|S\|}{c_{k}+b_{k} I_{\Phi}(x)}\right)^{1 / \alpha}\right)\right)\right]<\infty .
$$

Proof: With (A1) obviously

$$
\mathrm{P}\left(x \in \bigcap_{k=1}^{n} C(\ldots)\right) \leq \prod_{k=1}^{n} \mathbb{I}\left(x_{k} \in B\left(x, R^{*}\right)\right)
$$

and thus the integral in (5.12) is not bigger than $\left(\mu\left(B\left(x, R^{*}\right)\right)\right)^{n}$ which is finite. For (ii) observe that similarly to $(3.13)$ we have

$$
\mathbb{I}\left(x \in C\left(x_{k} ; \Phi+\sum_{i=1}^{n} \delta_{\left(x_{i}, Z_{k}\right)}\right)\right) \leq \mathbb{I}\left(x \in B\left(x_{k},\left(\beta \frac{a_{k}\left\|S_{k}\right\|}{c_{k}+b_{k} I_{\Phi}(x)}\right)\right)\right)
$$

and thus, changing the order of integration $\int \mathrm{E}[\ldots]=\mathrm{E}\left[\int \ldots\right]$ in $(5.12)$, we conclude by using the upper bound (5.16).

Corollary 5.6 If $\Phi$ is a homogeneous Poisson p.p. with intensity $\mu(d x)=\lambda d x$ and (A2) holds, then for (5.16) to be satisfied it is enough to assume that either (3.15) holds or (3.17) and the following condition are satisfied

$$
\int_{\mathbb{R}^{d}} e^{-\lambda \mathbf{b}_{d}|x|^{d}} \mathrm{E}\left[\left(L\left(S_{0}, x-y\right)\right)^{-n d / \alpha}\right] d y<\infty
$$


Remark: In order to calculate numerically the integral in (5.12), note that the probability $\mathrm{P}\left(x \in \bigcap_{k} C(\ldots)\right)$ can be expressed using the distribution function $F_{I_{\Phi}(x)}(\cdot)$ of the shot-noise process at $x$ in the following way (we assume for simplicity that $b>0$ a.s.)

$$
\mathrm{P}\left(x \in \bigcap_{k} C(\ldots)\right)=\mathrm{E}\left[F_{I_{\Phi}(x)}\left(\min _{1 \leq i \leq n}\left(\frac{a_{i}}{b_{i}} L\left(S_{i}, x-x_{i}\right)-\frac{c_{i}}{b_{i}}\right)-\sum_{k=1}^{n} L\left(S_{k}, x-x_{k}\right)\right)\right] .
$$

Knowing the distribution function $F_{I_{\Phi}(x)}(\cdot)$ (or its approximation) in a closed form, one can use a numerical integration procedure (for instance a monte-carlo method) to get (5.12). We remark that an effective method for the approximation of the shot-noise distribution (in dimension one and for a finite measure $\mu$ ) is presented in [7]; see also the references cited therein. Another possibility is to apply the contour integration technique suggested in the appendix.

\subsection{Volume fraction}

Volume fraction $p=\mathrm{P}(0 \in \Xi)$ is a basic characteristic of a stationary coverage process. Strictly speaking, it can be defined and calculated for any coverage process, but then the notion might be misleading, since it is only when we assume that the probability $\mathrm{P}(x \in \Xi)$ does not depend on $x$, that we can say that the expected fraction of the $d$-dimensional volume of $\Xi$ per unit ball is equal to $p$. Thus for the remaining part of this section we assume that $\Phi$ is a homogeneous Poisson p.p. with intensity $\lambda$ and that the function $L(s, x)$ is motion invariant in its second argument (i.e. it depends only on $(s,|x|))$. Using expansion (5.5) we can write

$$
p=\sum_{k=1}^{\infty} \frac{(-1)^{k+1}}{k !} \mathrm{E}\left[\left(N_{0}\right)^{(k)}\right]
$$

where the coefficients are given in Proposition 5.3, provided all moments are finite and the series is convergent. Note however, that if we assume condition (B) (that $b_{0} / a_{0} \geq \rho>0$ a.s.) then the expansion has finitely many non-zero terms only.

Remark: One can also try to calculate volume fraction via the following approach which is typical for the Boolean model:

$$
\begin{aligned}
p & \left.=1-\mathrm{E}\left[\prod_{i} \mathbb{I}\left(0 \notin C_{i}\right)\right)\right] \\
& =1-\mathrm{E}\left[\exp \left(\int_{\mathbb{R}^{d}} \log (\mathbb{I}(0 \notin C(x ; \Phi))) \Phi(d x)\right)\right] .
\end{aligned}
$$

However, the cell $C(x, \Phi)$ depends on the whole realization of the Poisson p.p., and not only on $(x, Z)$. Thus the expression in (5.20) does not have the form of the Laplace transform of the point process (as in the case of the Boolean model).

Nevertheless using the factorial moment expansion technique for a general class of functionals of spatial p.p. presented in [2] (see also papers cited there), the first order approximation of the volume fraction can be represented as

$$
\begin{aligned}
p & =\lambda \int_{\mathbb{R}^{d}} \mathrm{P}\left(0 \in C\left(x ; \delta_{(0, Z)}\right)\right) d x+O\left(\lambda^{2}\right) \\
& =\lambda \mathrm{E}\left[\nu_{d}\left(C\left(0 ; \delta_{(0, Z)}\right)\right)\right]+O\left(\lambda^{2}\right)
\end{aligned}
$$

RR $n^{\circ} 4019$ 
The first term in the last formula differs from the formula (5.14) for $\mathrm{E}\left[N_{0}\right]$ only in that $\Phi$ is replaced by the null measure (without points). More general polynomial (in $\lambda$ ) approximation formulas can be obtained from this expansion technique; note that the expansion formula proposed in (5.19) is not a polynomial in $\lambda$.

\section{From the Boolean model to the Poisson-Voronoi tessel- lation}

The aim of this section is to present the variety of patterns that one can obtain when playing with the model parameters. In particular, we investigate convergence results towards two limiting cases: the Boolean model and the Poisson Voronoi tessellation of the space. The convergence results are stated on the space of closed sets. They are complemented by differentiability results which lead to expansions for some key characteristics of the coverage process.

\subsection{Towards the Boolean model}

Note that the cells of $\Xi$ given by (2.1) are not mutually independent because of the presence of the shot-noise variable $I_{\Phi}$. However, if we assume $b=0$ a.s. ( $b \equiv 0$ for short), the cells are independent, and $\Xi$ is a Boolean model. In what follows, we study the following continuity problem: assume that $b \rightarrow 0$ in some sense. In what sense and under what conditions, does the typical cell $C(x, \Phi+\delta(x, Z))$ and the whole process $\Xi(\Phi)$ tend to their counterparts in the Boolean model obtained by assuming $b \equiv 0$ ? This is only a first step in the direction of the following more interesting differentiability question: assume the above continuity holds, and take $b$ small in some sense. What first order perturbation should one apply to the characteristics of the Boolean cells to get the characteristics of the dependent cells?

In the following we address this continuity question in two cases: (1) almost sure convergence of the model with $b$ scaled down by a constant $\epsilon \searrow 0$ and (2) weak convergence when $b \Rightarrow 0$ in distribution, before addressing the differentiability question.

\subsubsection{Convergence results}

Convergence of random sets For $\epsilon \geq 0$ let

$$
C_{i}^{(\epsilon)}=C_{i}^{(\epsilon)}(\Phi)=\left\{y: a_{i} L\left(S_{i}, y-X_{i}\right) \geq \epsilon b_{i} I_{\Phi}(y)+c_{i}\right\} .
$$

and $\Xi^{(\epsilon)}=\Xi^{(\epsilon)}(\Phi)=\bigcup_{i} C_{i}^{(\epsilon)}(\Phi)$. Note that $C_{i}^{(0)}$ does not depend on the whole $\Phi$ but $C_{i}^{(0)}(\Phi)=$ $C_{i}^{(0)}\left(\delta_{\left(X_{i}, Z\right)}\right)$ is the cell of a Boolean model $\Xi^{(0)}$.

In order to be able to prove an almost sure convergence theorem, we need the following technical condition for $L$ :

(C) for each $x \in \mathbb{R}^{d}$ and $s \in \mathbb{D}$, there exists a sequence $x_{n}$ such that $L\left(s, x_{n}\right)>L(s, x)$ and $\lim _{n} x_{n}=x$.

Proposition 6.1 Assume that the conditions of Proposition 3.2 part (ii) and Condition (C) are satisfied. For each point $X_{i} \in \Phi$, the following almost sure convergence holds on the space of closed sets $\lim _{\epsilon \rightarrow 0} C_{i}^{(\epsilon)}=C_{i}^{(0)}$. Moreover, a.s. and on the space of closed sets $\lim _{\epsilon \rightarrow 0} \Xi^{(\epsilon)}=\Xi^{(0)}$. 
Proof: For fixed $i$, the set $C_{i}^{(\epsilon)}$ is increasing in $\epsilon \rightarrow 0$. Thus its limit is equal to $\overline{\bigcup_{\epsilon} C_{i}^{(\epsilon)}}$, where $\bar{F}$ is the closure of $F$ (see e.g. [12] Cor. 3, p. 7). Obviously

$$
\overline{\bigcup_{\epsilon} C_{i}^{(\epsilon)}} \subset C_{i}^{(0)}
$$

because $C_{i}^{(\epsilon)} \subset C_{i}^{(0)}$ and $C_{i}^{(0)}$ is closed.

It remains to show that $C_{i}^{(0)} \subset \overline{\bigcup_{\epsilon} C_{i}^{(\epsilon)}}$. For this, (for each fixed $i$ ) take any $y \in C_{i}^{(0)}$. This means $a_{i} L\left(S_{i}, y-X_{i}\right) \geq c_{i}$. Note that if $a_{i}=0$ then the result trivially holds because by the assumption $c_{i}>0$ and thus $C_{i}^{(\epsilon)}=C_{i}^{(0)}=\emptyset$. So we now assume $a_{i}>0$. Condition (C) then guarantees the existence of a sequence $y_{n} \rightarrow y$ such that for all $n, a_{i} L\left(S_{i}, y_{n}-X_{i}\right)>c_{i}$, which implies that $y_{n} \in C_{i}^{\left(\epsilon_{n}\right)}$ for some $\epsilon_{n}>0$. So

$$
y=\lim _{n} y_{n} \in \overline{\bigcup_{n} C_{i}^{\left(\epsilon_{n}\right)}}
$$

For the second part of the theorem, observe that

$$
\overline{\bigcup_{\epsilon} \Xi(\epsilon)}=\overline{\bigcup_{\epsilon} \bigcup_{i} C_{i}^{(\epsilon)}}=\overline{\bigcup_{i} \overline{\bigcup_{\epsilon} C_{i}^{(\epsilon)}}}=\bigcup_{i} C_{i}^{(0)},
$$

because, under the conditions of Proposition 3.2, the union is closed.

Example 6.2 We now illustrate Proposition 6.1 by showing some patterns of our coverage process $\Xi$ "conforming" to a Boolean model pattern. We simulated a Poisson p.p. with 60 points on the square $[-5,15]^{2}$ (so that $\mu=0.15$ ). While observing only the square $[0,10]^{2}$, we take all 60 points of the larger square into account for evaluating $I_{\Phi}$. The response function is $L(s, y)=s(1+|y|)^{-3} ; S$ is uniformly distributed on $[0,2], a \equiv 1$ and $c \equiv 0.1$. The various patterns result from taking various deterministic values for $b$. Figure 1 presents the coverage process $\Xi$ "on its way" to a Boolean model. We have: a) $b \equiv 0.4$; note that $2 b<1<3 b$; thus at most 2 cells could cover any given point, although this is not observed; b) $b \equiv 0.2$; since $4 b<1=5 b$, at most 4 cells could cover any given point; c) $b \equiv 0.1$; cells occupy more and more of the final space that they occupy under the Boolean model regime; d) $b \equiv 0.0001$; almost the limiting case where each cell is a disk with independent radius distributed as $(10 S)^{1 / 3}-1$ (with mean $\left.20^{1 / 3} \cdot 3 / 4-1 \approx 1.035\right)$. On Figure 2 we have the same process but the limiting Boolean model has much larger grains, with radius $(5000 S)^{1 / 3}-1$ (with mean $\approx 16.15$ ). In the very "nested" pattern of b), some cells have "holes" around certain generating points.

Convergence of characteristics We can also prove the convergence of some characteristics of our scaled process to those of the Boolean model, including the probability for a point to be covered (volume fraction), the capacity functional, and the volume of the typical cell. This can only be done under some additional conditions, because these characteristics are not continuous functions on the space of closed sets. We first state an auxiliary lemma providing continuity conditions for certain indicator functions. For any fixed compact $K \subset \mathbb{R}^{d}, x \in \mathbb{R}^{d}, s \in \mathbb{D}$, $a, b \in \mathbb{R}^{+}, c>0, Z=(S,(a, b, c))$ and a function $f: \mathbb{R}^{d} \longrightarrow \mathbb{R}^{+}$denote

$$
\begin{aligned}
C^{(\epsilon)}(x, Z) & =\left\{y \in \mathbb{R}^{d}: a L(s, y-x) \geq \epsilon b f(y)+c\right\} \\
D(x, Z) & =\left\{y \in \mathbb{R}^{d}: a L(s, y-x)=c\right\} .
\end{aligned}
$$

$\mathrm{RR} \mathrm{n}^{\circ} 4019$ 


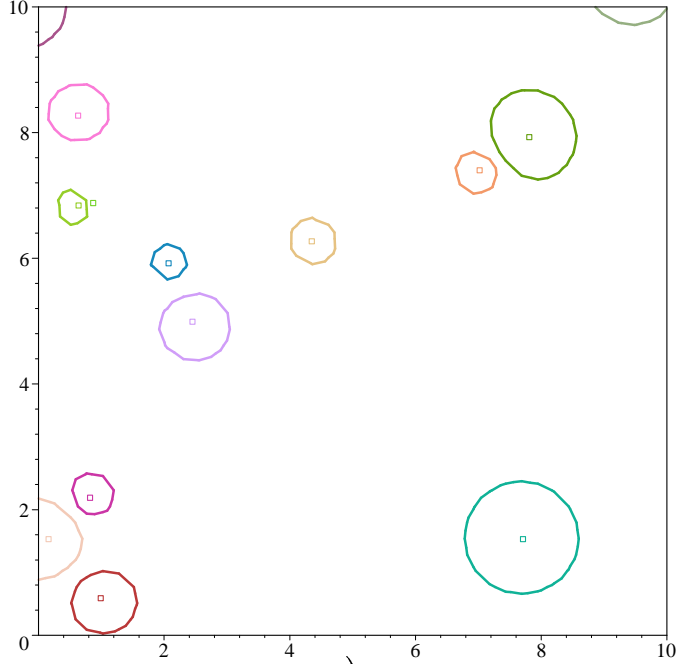

a)

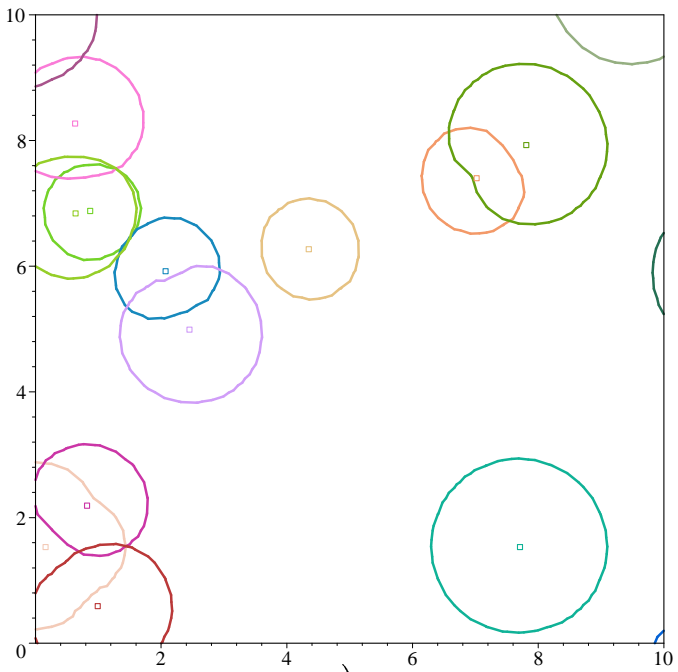

c)

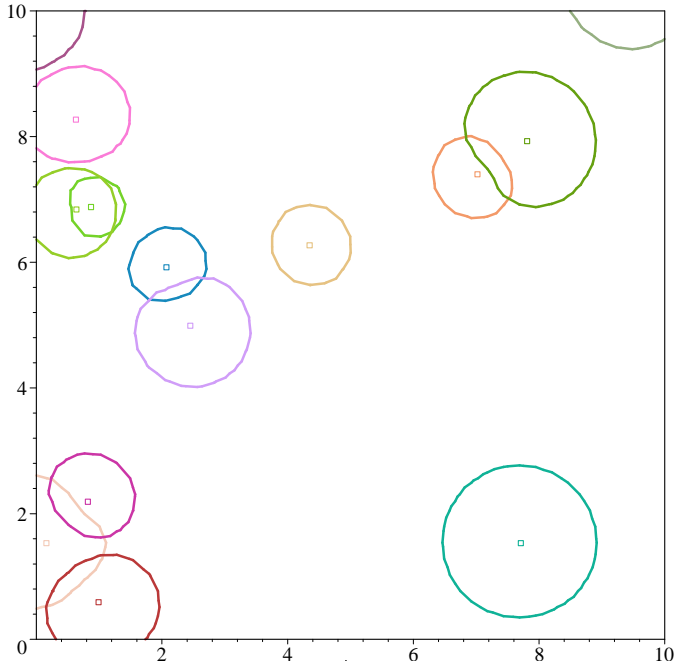

b)

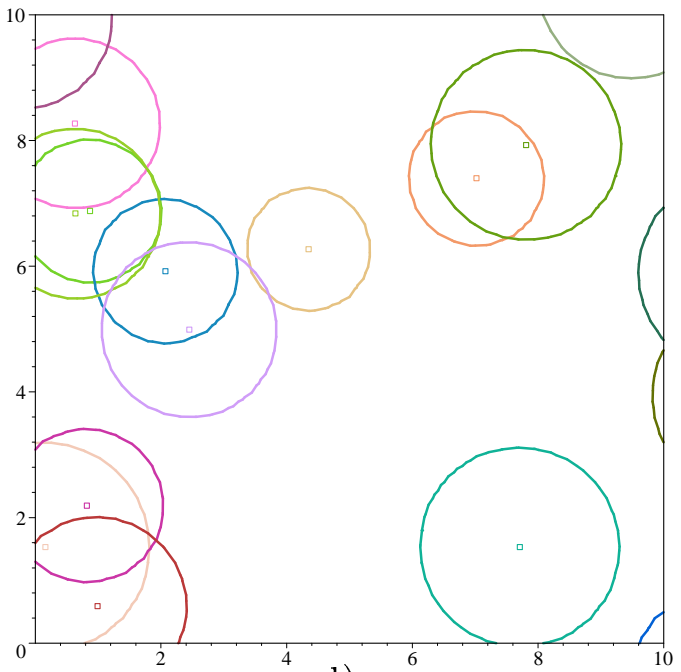

d)

Figure 1: The first coverage process of Example 6.2 "on its way" to a Boolean model.

Note that $D(x, Z)$ does not depend on $b$, whereas $C(x, Z)$ depends implicitly on the function $f$. We prefer however to keep this notation in order to have the following consistency between (6.1) for the typical cell located at $x$ and (6.2):

$$
C^{(\epsilon)}\left(x ; \Phi+\delta_{(x, Z)}\right)=C^{(\epsilon)}(x, Z)
$$

with $f(x)=I_{\Phi}(y)+L(s, y-x)$.

\section{Lemma 6.3 If}

$$
x \notin((\check{D}(0, Z) \oplus K) \backslash(\check{D}(0, Z) \oplus \stackrel{\circ}{K}))
$$

then

$$
\lim _{\epsilon \rightarrow 0} \mathbb{I}\left(K \cap C^{(\epsilon)}(x, Z) \neq \emptyset\right)=\mathbb{I}\left(K \cap C^{(0)}(x, Z) \neq \emptyset\right)
$$




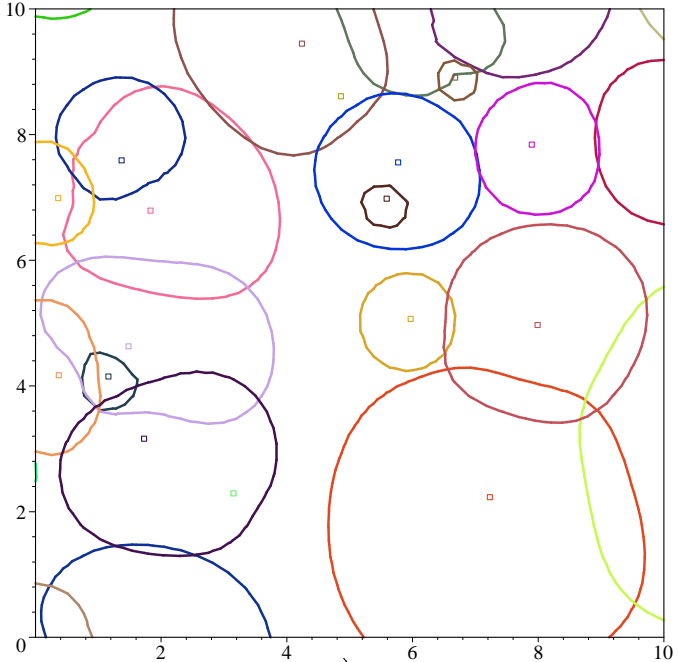

a)

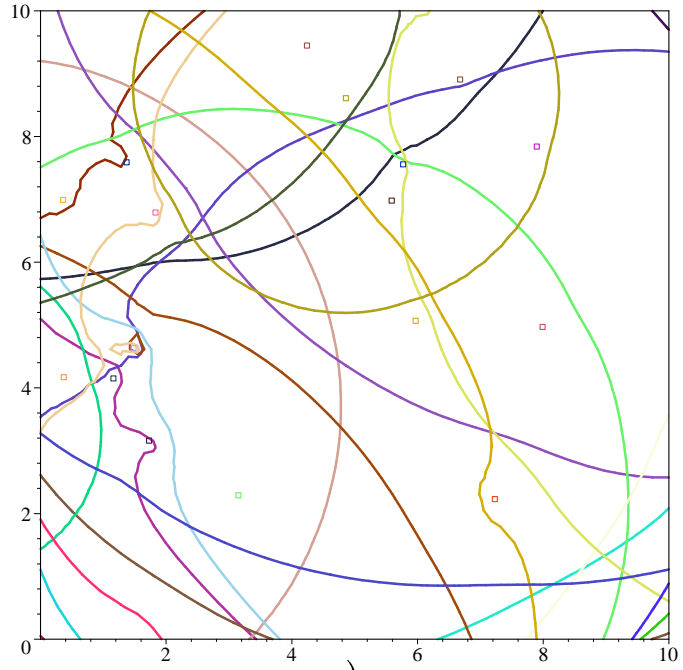

c)

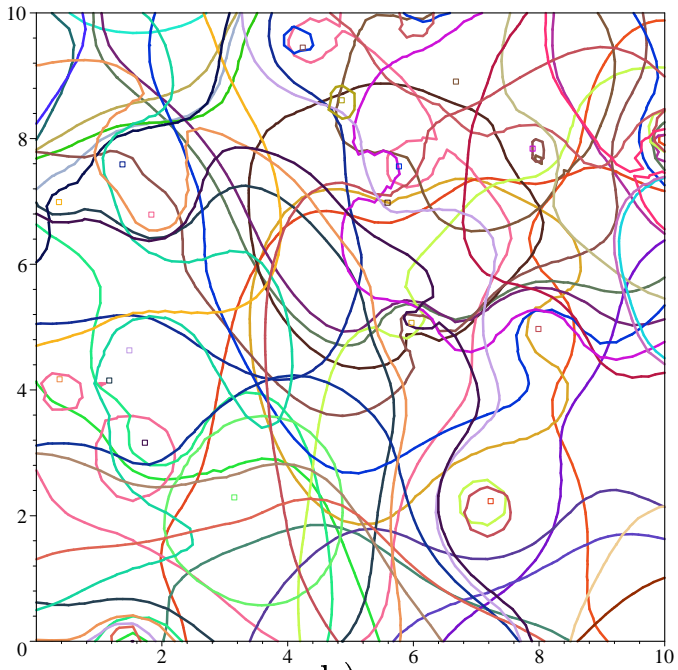

b)

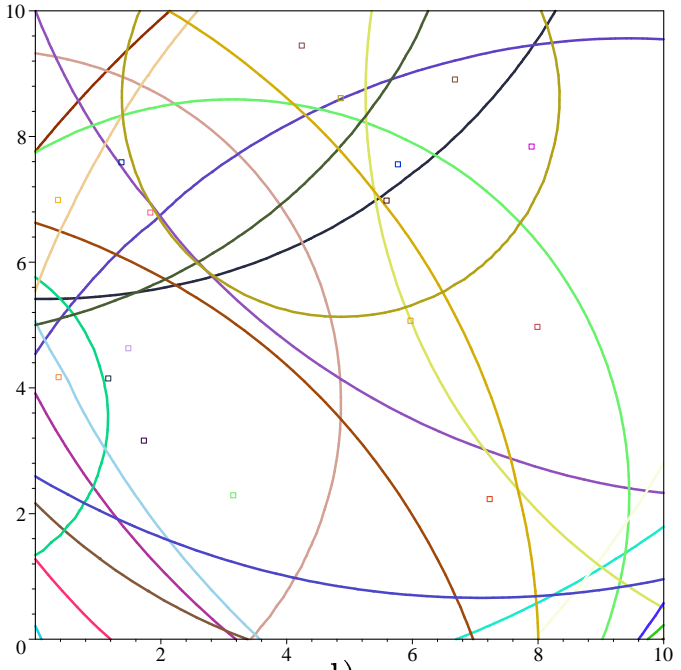

d)

Figure 2: The second coverage process of Example 6.2 "on its way" to a Boolean model.

Proof: (6.5) follows from the following inequalities

$$
\begin{aligned}
\mathbb{I}\left(K \cap C^{(0)}(x, Z) \neq \emptyset\right)-\mathbb{I} & (K \cap D(x, Z) \neq \emptyset, \stackrel{\circ}{K} \cap D(x, Z)=\emptyset) \\
\leq & \lim _{\epsilon \rightarrow 0} \mathbb{I}\left(K \cap C^{(\epsilon)}(x, Z) \neq \emptyset\right) \\
\leq & \mathbb{I}\left(K \cap C^{(0)}(x, Z) \neq \emptyset\right) .
\end{aligned}
$$

Inequality (6.7) is immediate from the fact that $C^{(\epsilon)}(x, Z) \subset C^{(0)}(x, Z)$ for $\epsilon \geq 0$.

In order to prove (6.6), it is enough to show that if $K \cap C^{(0)}(x, Z) \neq \emptyset$ and if in addition, for all $\epsilon>0, K \cap C^{(\epsilon)}(x, Z)=\emptyset$, then the second indicator in the left hand side of (6.6) is equal to 1. But under these two assumptions, there exists $z \in K$ such that $a L(s, z-x) \geq c$ and $a L(z-x)<c+\epsilon_{1}$ for any positive $\epsilon_{1}$, and so $a L(s, z-x)=c$. This means $K \cap D(x, Z) \neq \emptyset$. Assume now that also $\stackrel{\circ}{K} \cap D(x, Z) \neq \emptyset$ and let $y \in \stackrel{\circ}{K} \cap D(x, Z)$. Assume $a>0$; otherwise 
the result of the lemma is trivial, since $c>0$. By Condition $(\mathrm{C})$ we can find $y^{\prime} \in \stackrel{\circ}{K}$ in the neighborhood of $y$, such that $a L\left(s, y^{\prime}-x\right)>c$. This gives $K \cap C^{(\epsilon)}(x, Z) \neq \emptyset$ for some $\epsilon>0$, contradicting our assumption and concluding the proof of (6.6).

In order to complete the whole proof note that for any set $D \subset \mathbb{R}^{d} D \cap D(x, Z) \neq \emptyset$ iff $x \in(\check{D}(0, Z) \oplus D)$.

Capacity functional of the typical cell The following result is a straightforward consequence of Lemma 6.3.

Proposition 6.4 Suppose the conditions of Proposition 3.2 part (ii) and Condition (C) are satisfied. Let $K$ be a compact set and $x$ be a point of $\mathbb{R}^{d}$ such that

$$
\mathrm{P}(x \in((\check{D}(0, Z) \oplus K) \backslash(\check{D}(0, Z) \oplus \stackrel{\circ}{K})))=0
$$

where $Z=(S,(a, b, c))$ is a generic mark. Then we have convergence of the capacity functional of the typical cell on the set $K$

$$
\lim _{\epsilon \rightarrow 0} \mathrm{P}\left(K \cap C^{(\epsilon)}\left(x ; \Phi+\delta_{(x, Z)}\right) \neq \emptyset\right)=\mathrm{P}\left(K \cap C^{(0)}\left(x ; \delta_{(x, Z)}\right) \neq \emptyset\right) .
$$

Remark: By considering the simple example of a singleton $K=\{y\}$, one can see that Proposition 6.4 cannot be a straightforward application of the relationships between weak convergence and pointwise convergence of probability measures on continuity sets. Condition (6.8) reads

$$
\mathrm{P}(a L(S, y-x)=c)=0
$$

and it guarantees convergence of the probabilities on the set $\mathcal{F}_{\{y\}}=\{F$ closed $: y \in F\}$. The boundary of this set $\partial \mathcal{F}_{\{y\}}$ is equal to $\mathcal{F}_{\{y\}}$; thus for it to be a continuity set for the limiting Palm distribution of the typical cell of our Boolean model located at $x$ (i.e. the cell $\{y: a L(S, y-x) \geq c\})$, we need $\mathrm{P}(a L(S, y-x) \geq c)=0$, which is more restrictive than (6.10).

\section{Volume of the typical cell}

Proposition 6.5 If the conditions of Proposition 3.2 part (ii), condition (C) and (6.10) are satisfied for Lebesgue-a.s. all $y \in \mathbb{R}^{d}$, then the d-dimensional volume of the typical cell $C^{(\epsilon)}(x ; \Phi+$ $\left.\delta_{(x, Z)}\right)$ converges in distribution to the volume of $C^{(0)}\left(x ; \delta_{(x, Z)}\right)$.

Proof: We can express the volume of a cell as the following integral with respect to Lebesgue measure:

$$
\begin{aligned}
\nu_{d}\left(C^{(\epsilon)}\left(x ; \Phi+\delta_{(x, Z)}\right)\right) & =\int_{\mathbb{R}^{d}} \mathbb{I}\left(y \in C^{(\epsilon)}\left(x ; \Phi+\delta_{(x, Z)}\right)\right) d y \\
& =\int_{\mathbb{R}^{d}} \mathbb{I}\left(y \in C^{(\epsilon)}\left(x, \Phi+\delta_{(x, Z)}\right)\right) \mathbb{I}(a L(S, y-x) \neq c) d y
\end{aligned}
$$

where the second equality is a consequence of the assumption that (6.10) is satisfied for Lebesgue-a.s. all $y \in \mathbb{R}^{d}$. Lemma 6.3 and the monotone convergence theorem complete the proof. 


\section{Number of cells hitting a set}

Proposition 6.6 Suppose the conditions of Proposition 3.2 part (ii) and Condition (C) are satisfied. If for a given compact $K \in \mathbb{R}^{d}$

$$
\mathrm{E}[\mu((\check{D}(0, Z) \oplus K) \backslash(\check{D}(0, Z) \oplus \stackrel{\circ}{K}))]=0
$$

where $Z=(S,(a, b, c))$ is a generic mark, then the number of cells $N_{K}\left(\Xi^{(\epsilon)}\right)$ hitting set $K$ converges almost surely and in expectation to the number of cells of $\Xi^{(0)}$ hitting $K$, that is to a Poisson random variable with expectation $\mathrm{E}\left[\mu\left(\check{C}^{(0)}\left(0 ; \delta_{(0, Z)}\right) \oplus K\right)\right]$.

Proof:

$$
\lim _{\epsilon \rightarrow 0} N_{K}\left(\Xi^{(\epsilon)}\right)=\lim _{\epsilon \rightarrow 0} \sum_{i} \mathbb{I}\left(K \cap C_{i}^{(\epsilon)} \neq \emptyset\right)=\sum_{i} \mathbb{I}\left(K \cap C_{i}^{(0)} \neq \emptyset\right) .
$$

This follows from Lemma 6.3 under Assumption (6.11), which guarantees that the expected number of points $X_{i}$ of $\Phi$ not satisfying (6.4) is equal to 0 .

Corollary 6.7 Under the assumptions of Proposition 6.6, we have convergence of the capacity functional

$$
\lim _{\epsilon \rightarrow 0} \mathrm{P}\left(K \cap \Xi^{(\epsilon)} \neq \emptyset\right)=1-\exp \left[-\mathrm{E}\left[\mu\left(\check{C}^{(0)}\left(0 ; \delta_{(0, Z)}\right) \oplus K\right)\right]\right]
$$

Convergence in distribution Assume now a different scenario. Let us denote $G(d(a, b, c) \mid s)$ the conditional distributions of $A=(a, b, c)$ given $S=s$, where $Z=(S, A)$ is the generic mark. Suppose moreover that for each $s$ there is given a family $G_{\epsilon}(\epsilon \geq 0)$ of distributions satisfying $G_{\epsilon} \Rightarrow G_{0}$ weakly, and that under $G_{0}$, we have $b=0$ a.s. Let $\mathrm{P}^{(\epsilon)}$ be the probability referring to the model with the independently marked Poisson p.p. and where the distribution of the generic mark $Z$ is $G_{\epsilon}(d(a, b, c) \mid s) H(d s)$.

Proposition 6.8 Suppose the conditions of Proposition 3.2 part (ii) and Condition (C) are satisfied for all models with sufficiently small $\epsilon$. Let $K$ be a compact set and $x$ be a point of $\mathbb{R}^{d}$ such that condition (6.8) is satisfied with $\mathrm{P}$ replaced by $\mathrm{P}^{(0)}$. Then we have convergence of the capacity functional of the typical cell on the set $K$

$$
\lim _{\epsilon \rightarrow 0} \mathrm{P}^{(\epsilon)}\left(K \cap C\left(x ; \Phi+\delta_{(x, Z)}\right) \neq \emptyset\right)=\mathrm{P}^{(0)}\left(K \cap C\left(x ; \delta_{(x, Z)}\right) \neq \emptyset\right) .
$$

Proof:

$$
\begin{aligned}
\mathrm{P}^{(\epsilon)} & \left(K \cap C\left(x ; \Phi+\delta_{(x, Z)}\right) \neq \emptyset\right) \\
& =\iint_{\mathbb{D}} \int_{\left(\mathbb{R}^{+}\right)^{3}} \mathbb{I}\left(K \cap C\left(x ; \Phi+\delta_{(x,(s,(a, b, c)))} \neq \emptyset\right) G_{\epsilon}(d(a, b, c) \mid s) H(d s) \mathrm{P}(d \Phi),\right.
\end{aligned}
$$

where the outer integral is taken over the space of point processes with respect to the distribution of the marked Poison p.p. with $S$ distributed according to $H$. Now, by dominated convergence it is enough to prove that

$$
\left.\lim _{\epsilon \rightarrow 0} \int_{\left(\mathbb{R}^{+}\right)^{3}} \mathbb{I}(K \cap C(\ldots) \neq \emptyset) G_{\epsilon}(d(a, b, c) \mid s)=\int_{\left(\mathbb{R}^{+}\right)^{3}} \mathbb{I}(K \cap C(\ldots)) \neq \emptyset\right) G_{0}(d(a, b, c) \mid s)
$$

RR $n^{\circ} 4019$ 
for $H \times \mathrm{P}$ almost all $(s, \Phi)$. This will hold if the set $\left\{(a, b, c): a L(s, y-x) \geq b I_{\Phi}(y)+c\right.$ for some $y \in K\}$ is a continuity set for $G_{0}$. The boundary of this set is included in $\{(a, b, c)$ : $a L(s, y-x)=b I_{\Phi}(y)+c$ for some $y \in K$ and $a L(s, y-x) \leq b I_{\Phi}(y)+c$ for all $\left.y \in K\right\}$. As in the proof of Lemma 6.3, one can show under Condition $(\mathrm{C})$, that the restriction of this last set to $b=0$ (and $a>0)$ is contained in $\{(a, 0, c): a L(s, y-x)=c$ for some $y \in K$ and for no $y \in \stackrel{\circ}{K}\}$. This set is equal to

$$
\{(a, 0, c): x \notin((\check{D}(0, Z) \oplus K) \backslash(\check{D}(0, Z) \oplus \stackrel{\circ}{K})),
$$

where $Z=(a, 0, c)$. Under the assumptions of our proposition, this set has $G_{0}$ measure 0 for $H \times \mathrm{P}$ almost all $(s, \Phi)$. This completes the proof.

Corollary 6.9 If the conditions of Proposition 6.8 are satisfied for all compact $K$, then the typical cell converges in distribution on the space of closed sets.

\subsubsection{Perturbation formulas}

Coverage probability The setting is that of Section 6.1.1. Note that Proposition 6.4 with $K=\{y\}$ gives the following approximation of the probability $p_{x}^{(\epsilon)}(y)$ that the typical cell $C^{(\epsilon)}\left(x ; \Phi+\delta_{(x, Z)}\right)$ covers point $y$ :

$$
p_{x}^{(\epsilon)}(y)=\mathrm{P}(a L(S, y-x) \geq c)+o(1), \quad \epsilon \rightarrow 0,
$$

provided $\mathrm{P}(a L(S, y-x)=c)=0$. Now we briefly show how to derive the first and higher order expansions of this functional.

For simplicity, we restrict ourselves to the signal-to-interference-ratio model of Section 2.4 (although this approach can be generalized to other cases). Within this setting, taking $\kappa_{i}=\epsilon$ for all $i$, where $\epsilon$ is a constant, we can give a direct physical interpretation of these expansion formulas as the first and higher order corrections to be made on the coverage probability of the typical Boolean cell in order to take into account small interferences. In this case, let

$$
\tilde{p}_{x}^{(\epsilon)}(y)=\mathrm{P}\left(a L(S, y-x) \geq \epsilon b I_{\Phi}(y)+c\right) .
$$

Let $F_{*}$ denote the left-continuous version of the conditional distribution function of the random variable $(a L(S, y-x)-c) / b$ given $b>0$; i.e.,

$$
F_{*}(u)=\mathrm{P}\left(\frac{a L(S, y-x)-c}{b}<u \mid b>0\right) .
$$

We suppose that $F_{*}$ admits the following approximation at 0

$$
\lim _{u \searrow 0} \frac{F_{*}(u)-F_{*}(0)}{u^{\eta}}=f_{*} \text { for some } \eta \geq 0, f_{*}<\infty .
$$

Proposition 6.10 Assume that (6.17) holds for some $\eta \geq 0$ and $f_{*}<\infty$. Then

$$
\tilde{p}_{x}^{(\epsilon)}(y)=\mathrm{P}(a L(S, y-x) \geq c)-\epsilon^{\eta} f_{*} \mathrm{P}(b>0) \mathrm{E}\left[\left(I_{\Phi}(y)\right)^{\eta} \mathbb{I}\left(I_{\Phi}(y)>0\right)\right]+o\left(\epsilon^{\eta}\right),
$$

provided $\mathrm{E}\left[\left(I_{\Phi}(y)\right)^{\eta}<\infty\right]$. 
Remark: Note that if $(a L(S, y-x)-c) / b$ has an atom at 0; i.e., if $\mathrm{P}(s L(S, y-x)=c)>0$ (which is not possible under (6.10)), then (6.17) holds for $\eta=0, f_{*}=\mathrm{P}(s L(S, y-x)=c \mid b>0)$, and thus (6.18) yields

$$
\begin{aligned}
\tilde{p}_{x}^{(\epsilon)}(y) & =\mathrm{P}(a L(S, y-x) \geq c)-\mathrm{P}\left(a L(S, y-x)=c, I_{\Phi}(y)>0\right)+o(1) \\
& =\mathrm{P}(a L(S, y-x)>c)+\mathrm{P}\left(a L(S, y-x)=c, I_{\Phi}(y)=0\right)+o(1) .
\end{aligned}
$$

Another typical case would be when $F_{*}(u)$ admits the density $f_{*}(u)$ that is finite at the origin; then $\eta=1$ and $f_{*}=f_{*}(0)$.

Proof or Proposition 6.10: We have

$$
\tilde{p}_{x}^{(\epsilon)}(y)=\mathrm{P}(a L(S, y-x) \geq c)-\mathrm{P}\left(0 \leq \frac{a L(S, y-x)-c}{b}<\epsilon I_{\Phi}(y) \mid b>0\right) \mathrm{P}(b>0) .
$$

Since $Z=(S,(a, b, c))$ and $I_{\Phi}$ are independent

$$
\mathrm{P}\left(0 \leq \frac{a L(S, y-x)-c}{b}<\epsilon I_{\Phi}(y) \mid b>0\right)=\mathrm{E}\left[F_{*}\left(\epsilon I_{\Phi}(y)\right)-F_{*}(0)\right] .
$$

If $\mathrm{E}\left[\left(I_{\Phi}(y)\right)^{\eta}\right]<\infty$ and $(6.17)$ holds then

$$
\mathrm{E}\left[\frac{F_{*}\left(\epsilon I_{\Phi}(y)\right)-F_{*}(0)}{\left(\epsilon I_{\Phi}(y)\right)^{\eta}} \mathbb{I}\left(I_{\Phi}(y)>0\right)\left(I_{\Phi}(y)\right)^{\eta}\right] \leq \mathrm{E}\left[\left(f_{*}+M\right)\left(I_{\Phi}(y)\right)^{\eta}\right]<\infty
$$

for some constant $M<\infty$ and all $\epsilon>0$, and thus by the dominated convergence theorem

$$
\begin{aligned}
& \lim _{\epsilon \rightarrow 0} \frac{1}{\epsilon^{\eta}} \mathrm{P}\left(0 \leq \frac{a L(S, y-x)-c}{b}<\epsilon I_{\Phi}(y) \mid b>0\right) \\
& \quad=\mathrm{E}\left[\lim _{\epsilon \rightarrow 0} \frac{F_{*}\left(\epsilon I_{\Phi}(y)\right)-F_{*}(0)}{\left(\epsilon I_{\Phi}(y)\right)^{\eta}} \mathbb{I}\left(I_{\Phi}(y)>0\right)\left(I_{\Phi}(y)\right)^{\eta}\right] \mathrm{P}(b>0) \\
& \quad=f_{*} \mathrm{P}(b>0) \mathrm{E}\left[\left(I_{\Phi}(y)\right)^{\eta} \mathbb{I}\left(I_{\Phi}(y)>0\right)\right],
\end{aligned}
$$

which completes the proof.

If the distribution function $F_{*}$ admits a higher order approximation then we can give a higher order approximation of $p_{x}^{(\epsilon)}(y)$. Here we briefly state the result assuming that $F_{*}$ has $h$ derivatives $F_{*}^{(k)}(0), k=1, \ldots, h$, at 0 ; i.e.,

$$
F_{*}(u)=F_{*}(0)+\sum_{k=1}^{h} \frac{F_{*}^{(k)}(0)}{k !} u^{k}+\mathcal{R}(u) \quad \text { and } \quad \mathcal{R}(u)=o\left(u^{h}\right) \quad u \searrow 0 .
$$

Proposition 6.11 Assume that (6.20) holds for some $h \geq 1$. Then

$$
\tilde{p}_{x}^{(\epsilon)}(y)=\mathrm{P}(a L(S, y-x) \geq c)-\mathrm{P}(b>0) \sum_{k=1}^{h} \epsilon^{k} \frac{F_{*}^{(k)}(0)}{k !} \mathrm{E}\left[\left(I_{\Phi}(y)\right)^{k}\right]+o\left(\epsilon^{h}\right),
$$

provided $\mathrm{E}\left[\left(I_{\Phi}(y)\right)^{h}\right]<\infty$.

The proof goes along the same lines as the proof of Proposition 6.10.

RR $n^{\circ} 4019$ 
Mean volume of the typical cell From (4.4) we see that, in principle, any approximation of the coverage probability also yields an approximation of the mean volume of the typical cell, simply by integration of the terms of the latter expansion with respect to $y$. In what follows we show how to justify the interchange of the integral and the expansion for the case of formula (6.21), assuming for simplicity that the Poisson p.p. is homogeneous and that $L(s, z)$ depends only on $s$ and $|z|$. In this case, we have the same distribution of $I_{\Phi}(y)$ for all $y$ and $\tilde{p}_{x}(y)$ only depends on $x-y$.

Let $x=0$ and

$$
\tilde{v}^{(\epsilon)}=\tilde{v}_{0}^{(\epsilon)}=\mathrm{E}\left[\nu_{d}\left(\left\{y: a L(S, y) \geq \epsilon b I_{\Phi}(y)+c\right\}\right)\right]
$$

In order to express the dependence on $y$, we will write $F_{*}(u ; y)$ and $F_{*}^{(k)}(u ; y)$ to denote $F_{*}$ defined in (6.16) and its derivatives with respect to $u$. Similarly, we will denote the remainder term in (6.20) by $\mathcal{R}(u ; y)$. Assume now that (6.20) holds for all $y \in \mathbb{R}^{d}$ and moreover

$$
|\mathcal{R}(u, y)| \leq \mathcal{H}_{1}(u) \mathcal{H}_{2}(y)
$$

where $\mathcal{H}_{1}(u)$ is a nondecreasing function satisfying

$$
\lim _{u \searrow 0} \frac{\mathcal{H}_{1}(u)}{u^{h}}=0
$$

and

$$
\int_{0}^{\infty} \mathcal{H}_{2}(y) d y<\infty .
$$

Proposition 6.12 Assume that (6.20) and (6.23)-(6.25) hold for some $h \geq 1$. Then

$$
\tilde{v}^{(\epsilon)}=\tilde{v}^{(0)}-\mathrm{P}(b>0) \sum_{k=1}^{h} \epsilon^{k} \frac{1}{k !} \int_{0}^{\infty} F_{*}^{(k)}(0 ; y) d y \mathrm{E}\left[\left(I_{\Phi}(0)\right)^{k}\right]+o\left(\epsilon^{h}\right),
$$

provided $\int_{0}^{\infty} F_{*}^{(k)}(0 ; y) d y<\infty$ for $k=1, \ldots, h$ and

$$
\mathrm{E}\left[\mathcal{H}_{1}\left(I_{\Phi}(0)\right)\left(I_{\Phi}(0)\right)^{h}\right]<\infty .
$$

Proof: By (4.4), (6.19) and (6.20) it suffices to show that

$$
\lim _{\epsilon \rightarrow 0} \epsilon^{-h} \int_{0}^{\infty} \mathrm{E}\left[\mathcal{R}\left(\epsilon I_{\Phi}(y) ; y\right)\right] d y=0
$$

For fixed $y$, by Proposition 6.21 we have pointwise convergence $\epsilon^{-h} \mathrm{E}[\ldots] \rightarrow 0$. We will establish the conditions of the dominated convergence theorem for $\lim \int \epsilon^{-h} \mathrm{E}[\ldots] d y$. For this, thanks to $(6.24)$, take any $\Delta>0$ and $u_{0}$ such that $\mathcal{H}_{1}(u) \leq \Delta$ for $u \leq u_{0}$. Now, by monotonicity of $\mathcal{H}_{1}(u)$, for $\epsilon \leq 1$

$$
\begin{aligned}
\epsilon^{-h} & \int_{0}^{\infty} \mathrm{E}\left|\mathcal{R}\left(\epsilon I_{\Phi}(y) ; y\right)\right| d y \\
& \leq \int_{0}^{\infty} \mathcal{H}_{2}(y) d y\left(\mathrm{E}\left[\Delta\left(I_{\Phi}(0)\right)^{h} \mathbb{I}\left(\epsilon I_{\Phi}(0) \leq u_{0}\right)\right]+\mathrm{E}\left[\mathcal{H}_{1}\left(I_{\Phi}(0)\right)\left(\frac{I_{\Phi}(0)}{u_{0}}\right)^{h} \mathbb{I}\left(\epsilon I_{\Phi}(0)>u_{0}\right)\right]\right)
\end{aligned}
$$

which is finite by (6.25) and the assumption (6.27); this completes the proof. 
Example 6.13 Consider the case where $\Phi$ is homogeneous Poisson p.p. on $\mathbb{R}^{d}, L(s, z)=\operatorname{sl}(z)$ and assume $S \in \mathbb{R}^{+}$admits a density $f_{S}$, and is independent of $a, b, c$. Then the conditions of Proposition 6.10 are satisfied if

$$
f_{*}=\mathrm{E}\left[b f_{S}\left(\frac{c}{a l(y-x)}\right) \mid b>0\right]<\infty .
$$

Assume in addition that $a, b$ and $c$ are strictly positive a.s. and that $l(z)=(1+|z|)^{-\alpha}$, with $\alpha>d$. Direct computations give the following first order expansion for the mean volume of the typical cell (provided we have finiteness of the moments which show up in the following expression):

$$
\begin{aligned}
\tilde{v}^{(\epsilon)}= & \frac{\mathbf{s}_{d}}{d} \mathrm{E}\left[\left(\left(\frac{a S}{c}\right)^{1 / \alpha}-1\right)_{+}^{d}\right] \\
& +\epsilon \frac{\mathbf{s}_{d} \mathrm{E}\left[I_{\Phi}(0)\right]}{\alpha} \sum_{k=0}^{d-1}\left(\begin{array}{c}
d-1 \\
k
\end{array}\right)(-1)^{d-1-k} \mathrm{E}\left[b\left(\frac{a}{c}\right)^{k+1 / \alpha}\right] \mathrm{E}\left[S^{k-1+1 / \alpha}\right]+o(\epsilon),
\end{aligned}
$$

where $\mathbf{s}_{d}$ is the $d-1$ dimensional area of the unit sphere in $\mathbb{R}^{d}$ and $(w)_{+}=\max (0, w)$. Note that the existence of the negative moment $\mathrm{E}\left[S^{-1+1 / \alpha}\right]$ is guaranteed by Condition (6.17).

Example 6.14 We continue with the model described in Example 4.1. Note that under the parameterization $a=1-\epsilon$ and $b=\epsilon$, we have $p_{x}(y)=\tilde{p}_{x}^{(\epsilon)}(y)$. The case $\epsilon=0$ refers to the Boolean model. We assume $S$ exponential with parameter $m$ and thus the coverage probability for the Boolean model (i.e. the constant in the expansion (6.21)) is equal to $\mathrm{E}\left[e^{-m c /(a l(y-x)}\right]=e^{-m / l(y-x)}$ for $a \equiv c \equiv 1$. The distribution function $F_{*}$ for $u \geq 0$ is $F_{*}(u)=1-\mathrm{E}\left[e^{-m(b u+c) /(a l(y-x))}\right]=1-e^{-m(u+1) / l(y-x)}$ (when $b \equiv 1$ ). Its first derivative at 0 is $m / l(y-x) e^{-m / l(y-x)}$. Moreover, the first moment of the shot noise with response function $l(y)=(\max (R,|y|))^{-4}$ is $\mathrm{E}\left[I_{\Phi}(y)\right]=2 \pi /\left(R^{2} m\right)=2 \pi / m$ (for $R=1$ ). Thus the first order perturbation formula for $p_{0}(y)$ is

$$
\tilde{p}_{0}(y) \approx e^{-m / l(y)}+\epsilon 2 \pi \frac{m}{l(y)} e^{-m / l(y)} .
$$

On Figure 3 (a) we compare the exact values of $p_{0}(1)$ calculated (for various $\epsilon$ ) using formula (A.1), with the first, second, 14-th and 15-th order approximation of $\tilde{p}^{(\epsilon)}(1)=\tilde{p}_{0}^{(\epsilon)}(1)$ given by (6.21) with $h=1,2,14,15$. Plot (b) shows the same order approximations of $\tilde{v}^{(\epsilon)}$ given by (6.26). We remark that the remainder term in (6.20) can be expressed in Lagrange's form as $\mathcal{R}(u ; y)=F_{*}^{(h+1)}\left(\theta_{y} u ; y\right) u^{h+1} /(h+1)$ !, with $0 \leq \theta_{y} \leq 1$, and we can take $\mathcal{H}_{1}(u)=m^{h+1} /(h+1) ! u^{(h+1)}$ and $\mathcal{H}_{2}(y)=(l(y))^{-h-1} e^{-m / l(y)}$ satisfying (6.23)-(6.25).

Mean number of cells covering a point In the homogeneous case, we can use the expansion for the mean volume of the typical cell and Relation (5.14) to derive expansions for the mean number of cells covering a point.

\subsection{Towards the Poisson-Voronoi tessellation}

The Voronoi tessellation generated by an (unmarked) Poisson p.p. $\Phi=\left\{X_{i}\right\}$ with $\mu\left(\mathbb{R}^{d}\right)=\infty$ (thus having infinite number of points) is the collection of cells $V\left(X_{i}\right)=V\left(X_{i} ; \Phi\right)$, where

$$
V\left(X_{i}\right)=\left\{y \in \mathbb{R}^{d}:\left|y-X_{i}\right| \leq \inf _{X_{k}: X_{k} \in \Phi-\delta_{X_{i}}}\left|y-X_{k}\right|\right\}
$$

RR $n^{\circ} 4019$ 

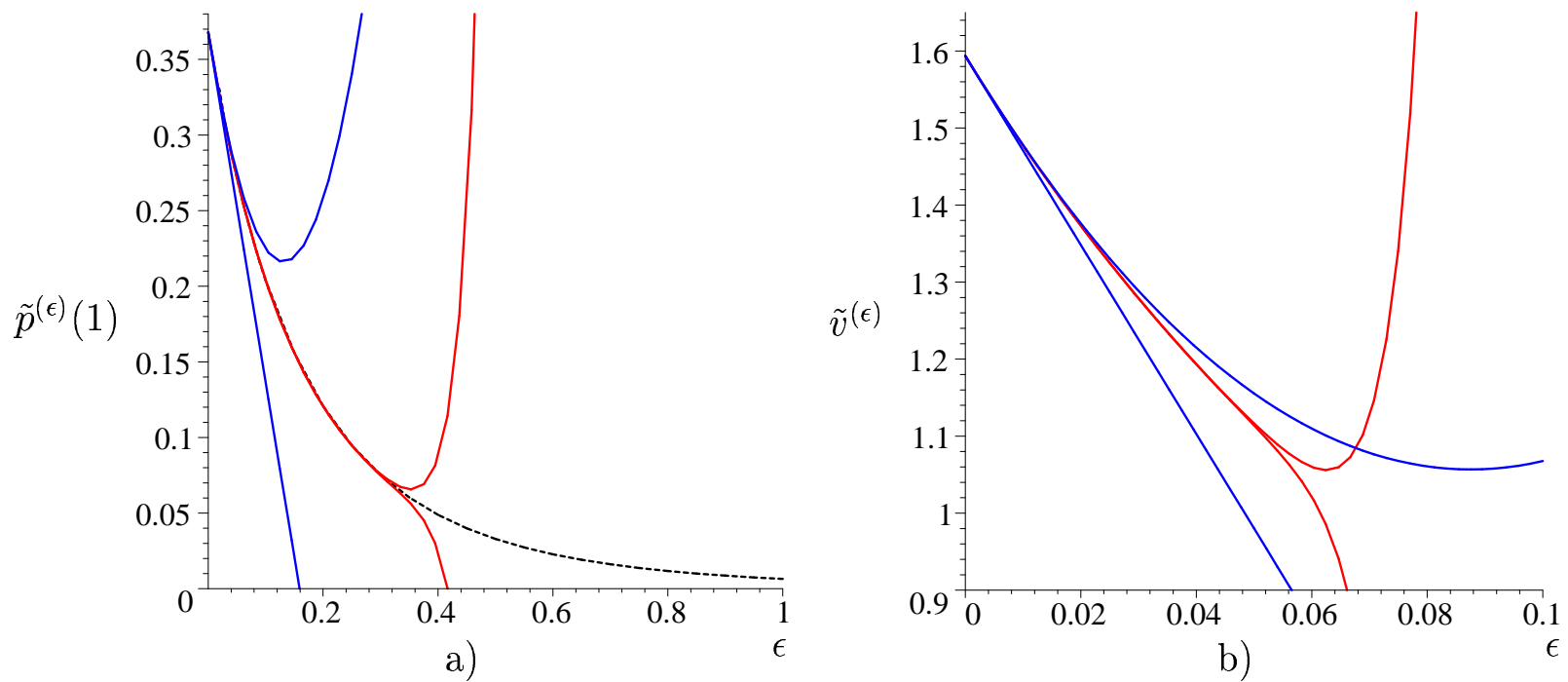

Figure 3: a) Exact values of $p_{x}(y)$ (dashed line, obtained from the singular integral representation) and the first, second, 14-th and 15-th order approximation of $\tilde{p}_{x}^{(\epsilon)}(y)$. b) Similar approximation for the mean area of the typical cell $\tilde{v}^{(\epsilon)}$ given by (6.26) (see Example 6.14)

From the above formula we see that the form of the cell attached to point $X_{i}$ is determined by some "neighboring" points of $X_{i}$ only. It is quite reasonable to expect that if we let the attenuation function $L(s, z)$ decrease fast in $z$, we will get the same effect. We will formalize this observation assuming $L(s, z)=s l(z)$ and taking appropriate families of $l$-functions (we will mainly concentrate on the class of functions which are used to represent Hertzian propagation, although more general cases can be handled in the same way).

Proposition 6.15 Let $l_{n}(z)=(1+|z|)^{-n}, c=0$ almost surely and let the conditions in Proposition 3.2 part (iii) be satisfied. Suppose moreover that $\mu\left(\mathbb{R}^{d}\right)=\infty$. Then for each point $X_{i} \in \Phi$ the following almost sure convergence of its cell holds on the space of closed sets

$$
\lim _{n \rightarrow \infty} C^{n}\left(X_{i} ; \Phi\right)=V\left(X_{i} ; \Phi\right)
$$

where $C^{n}(.$.$) is the cell associated with the attenuation function l_{n}$.

Proof: Fix a point of $\Phi$, without loss of generality call it $X_{0}$. Let $C^{n}=C^{n}\left(X_{0}\right.$, $\left.\Phi\right)$ and denote $I_{\Phi}^{n}(y)$ the shot-noise process with the attenuation function $l_{n}(z)=(1+|z|)^{-n}$. Note that under the assumption $\mu\left(\mathbb{R}^{d}\right)=\infty$ we have $I_{\Phi}^{n}(y)>0$ for all $n$. Moreover, since we have assumed $S>0$ almost surely (implied by part (iii) of Proposition 3.2), then

$$
\begin{aligned}
\left(I_{\Phi}(y)\right)^{1 / n}=\left(\sum_{X_{k} \in \Phi} S_{k}\left(1+\left|y-X_{k}\right|\right)^{-n}\right)^{1 / n} & \stackrel{n \rightarrow \infty}{\longrightarrow} \sup _{X_{k} \in \Phi}\left(1+\left|y-X_{k}\right|\right)^{-1} \\
& =\left(1+\min _{X_{k} \in \Phi}\left|y-X_{k}\right|\right)^{-1}
\end{aligned}
$$

(this property differs form the standard calculus exercise in that the number of terms in the sum is infinite; it uses the property that a.s. the above supremum is reached by a unique point of $\Phi$ ). 
Moreover the convergence is locally uniform in $y$. Note now that $C^{n}=\left\{y:\left|y-X_{0}\right| \leq f_{n}(y)\right\}$, where

$$
f_{n}(y)=\left(\frac{a_{0} S_{0}}{b_{0} I_{\Phi}(y)}\right)^{1 / n}-1
$$

By (6.30)

$$
\lim _{n \rightarrow \infty} f_{n}(y)=\min _{X_{k} \in \Phi}\left|y-X_{k}\right|
$$

locally uniformly in $y$. We now prove that $\lim _{n} C^{n}=\left\{y:\left|y-X_{0}\right| \leq \min _{X_{k} \in \Phi}\left|y-X_{k}\right|\right\}$. It is equivalent to proving that the following two conditions hold (see [12], Th. 1-2-2, p. 6).

(i) For any $y$ s.t. $\left|y-X_{0}\right| \leq \min _{X_{k} \in \Phi}\left|y-X_{k}\right|$, there exists a sequence of points $y_{n} \rightarrow y$ such that $\left|y_{n}-X_{0}\right| \leq f_{n}\left(y_{n}\right)$ for all sufficiently large $n$.

(ii) If a sequence of points $y_{k_{n}}$, such that $\left|y_{k_{n}}-X_{0}\right| \leq f_{k_{n}}\left(y_{k_{n}}\right)$ for all $n$, converges to $y$, then $\left|y-X_{0}\right| \leq \min _{X_{k} \in \Phi}\left|y-X_{k}\right|$.

In fact:

- ad (i) Suppose $y$ is in the interior of the Voronoi cell; i.e., $\left|y-X_{0}\right|<\min _{X_{k} \in \Phi}\left|y-X_{k}\right|$. Then $\left|y-X_{0}\right| \leq f_{n}(y)$ for all sufficiently large $n$ because $f_{n}(y) \rightarrow \min _{X_{k} \in \Phi}\left|y-X_{k}\right|$. So Condition (i) is satisfied with the constant sequence $y_{n}=y$. If $y$ is on the boundary of the Voronoi cell; i.e., $\left|y-X_{0}\right|=\min _{X_{k} \in \Phi}\left|y-X_{k}\right|$, then there exists a sequence of points $y_{n}$ converging to $y$ and such that for all $n,\left|y_{n}-X_{0}\right|<\min _{X_{k} \in \Phi}\left|y_{n}-X_{k}\right|$. One can use this sequence to construct the one required in (i).

- ad (ii) Let $y_{k_{n}}$ be as mentioned there. For all $n$

$$
\left|y_{k_{n}}-X_{0}\right| \leq f_{k_{n}}\left(y_{k_{n}}\right)
$$

Letting with $n \rightarrow \infty$ the left hand side tends to $\left|y-X_{0}\right|$ and the right hand side (because of the uniform convergence of $\left.f_{n}\right)$ to $\min _{X_{k} \in \Phi}\left|y-X_{k}\right|$ and we get $\left|y-X_{0}\right| \leq \min _{X_{k} \in \Phi}\left|y-X_{k}\right|$.

Remark: Obviously $\lim _{n} \Xi(\Phi) \rightarrow \mathbb{R}^{d}$. Moreover, if $c>0$ then almost surely

$$
\lim _{n \rightarrow \infty} C\left(X_{i} ; \Phi\right)=\left\{X_{i}\right\} \quad \text { (a single point). }
$$

We remark also that a result similar to (6.15) could be proven for any family of attenuation functions $l_{\alpha}$ satisfying

$$
\lim _{\alpha \rightarrow \alpha_{0}} l_{\alpha}^{-1}\left(\sum_{i} s_{i} l_{\alpha}\left(y_{i}\right)\right) \rightarrow \min _{i} y_{i}
$$

for any (positive) coefficients $s_{i}$. For example for $l_{\alpha}\left(y_{i}\right)=\exp \left[-\alpha y_{i}\right]$ and $\alpha_{0}=\infty$.

As previously, one can prove the convergence of various functionals. We consider here only the volume of the typical cell.

Proposition 6.16 The volume of the typical cell $C^{n}\left(x ; \Phi+\delta_{(x, z)}\right)$ converges in distribution to the volume of the typical cell $V\left(x ; \Phi+\delta_{(x, Z)}\right)$ of the Voronoi tessellation.

RR $n^{\circ} 4019$ 
Proof: This can be done using the following inequalities

$$
\begin{aligned}
\mathbb{I}(z \in V(x))-\mathbb{I}\left(|z-x|=\min _{X_{k} \in \Phi}\left|z-X_{k}\right|\right) & \leq \liminf _{n \rightarrow \infty} \mathbb{I}\left(z \in C^{n}\left(x, \Phi+\delta_{(x, Z)}\right)\right) \\
& \leq \limsup _{n \rightarrow \infty} \mathbb{I}\left(z \in C^{n}\left(x, \Phi+\delta_{(x, Z)}\right)\right) \\
& \leq \mathbb{I}(z \in V(x)),
\end{aligned}
$$

which hold for all $z \in \mathbb{R}^{d}$. Then, representing volumes as integrals with respect to Lebesgue measure, using Fatou lemmas for lim inf and limsup, we get the conclusion provided that

$$
\int_{\mathbb{R}^{d}} \mathbb{I}\left(|z-x|=\min _{X_{k} \in \Phi}\left|z-X_{k}\right|\right) d z=0
$$

almost surely. This is true for the Poisson p.p. $\Phi$ with a diffuse intensity measure $\mu$.

Example 6.17 We now illustrate Proposition 6.15 by showing some patterns of our coverage process $\Xi$ "conforming" to the Voronoi tessellation of the plane (see Figure 4). The Poisson p.p., the observation and the simulation windows are as in Example 6.2. $S$ is uniformly distributed on $[0,2], a \equiv 1, c \equiv 0, b \equiv 0.2$ thus allowing for overlapping of at most 4 cells for a given point. The various patterns result from taking the attenuation function $l(y)=(1+|y|)^{-\alpha}$ with various $\alpha$. We have: a) $\alpha=3$, b) $\alpha=5$, c) $\alpha=12$, d) $\alpha=100$. The effect of overlapping is still visible. A more accurate tessellation can be obtained inhibiting overlapping, e.g. by taking $b \equiv 0.5$ (see Figure $5 \mathrm{~d}$ ).

Relation to the Johnson-Mehl model We also have convergence to intermediate states of the Johnson-Mehl grain growth model (see e.g. [17], s. 10.7, p. 333-334).

Corollary 6.18 Under the assumptions of Proposition 6.15, if, instead of $c \equiv 0$, we take $c=b(R+1)^{-n}$ for some fixed or random variable $R$, then

$$
\lim _{n \rightarrow \infty} C^{n}\left(X_{i}, \Phi\right)=V\left(X_{i} ; \Phi\right) \cap B\left(X_{i}, R\right)
$$

Example 6.19 We now illustrate Corollary 6.18 by showing some patterns of our coverage process $\Xi$ "growing" to the Voronoi tessellation as in the Johnson-Mehl model (see Figure 5). The observation and simulation windows and the Poisson p.p. are as in the previous examples. $S$ is uniformly distributed on $[0,2]$ and we take $a \equiv 1$ and $b \equiv 0.5$, thus inhibiting any intersections. The attenuation function $l(y)=(1+|y|)^{-30}$ is strong enough to give a tessellation covering almost the whole plane when $c \equiv 0$. If we assume however $c=b(1+R)^{-30}$ and take: a) $R=0.4$, b) $R=1.2$, c) $R=2$, d) $R=\infty(c \equiv 0)$. As the result we get a sequence of sub- tessellations, with each of its cells constrained to a disk of radius $R$ (wherever a cell has diameter less than $R$ it has its final shape). All cells start growing at the same time. But the effect of a "late-comer" can also be modeled. On Figure 6 an extra point is added at the location $X=(5,3)$ at the time when the original tessellation has already "age" (size) $R=1.2$. The mark $S$ is taken to be

$$
S=\left(\frac{1+R-1.2}{1+R}\right)^{30}
$$

Note that for a point $y$ to be covered by this new cell, it has to have a distance from $X$ less 


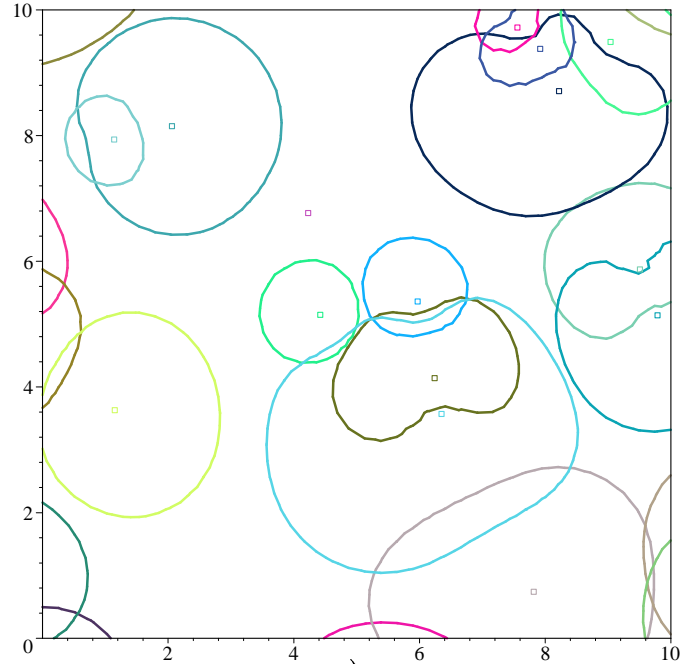

a)

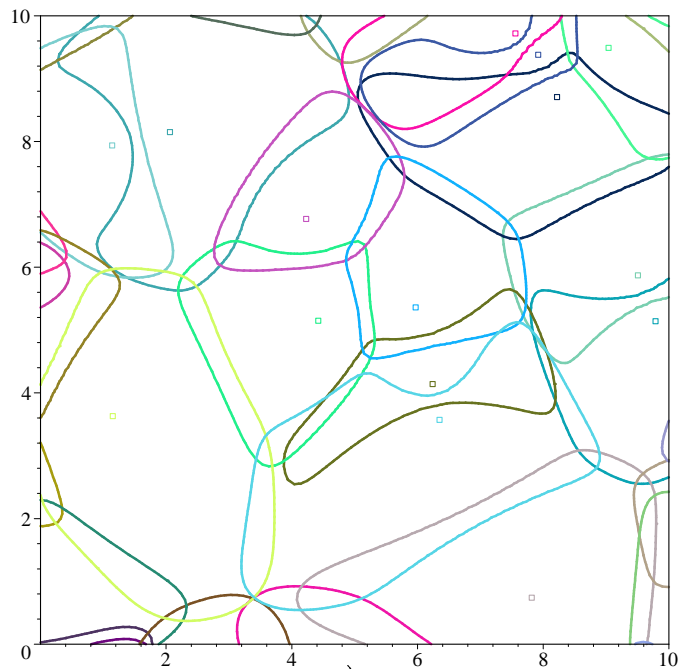

c)

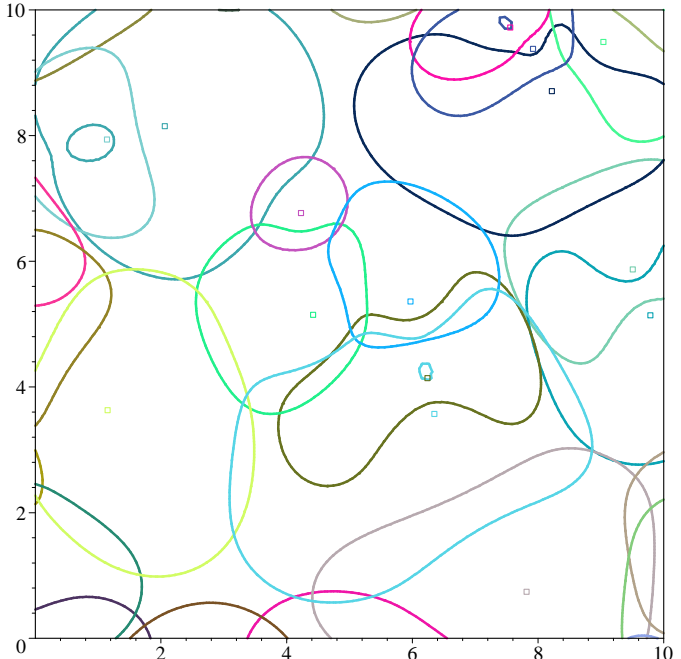

b)

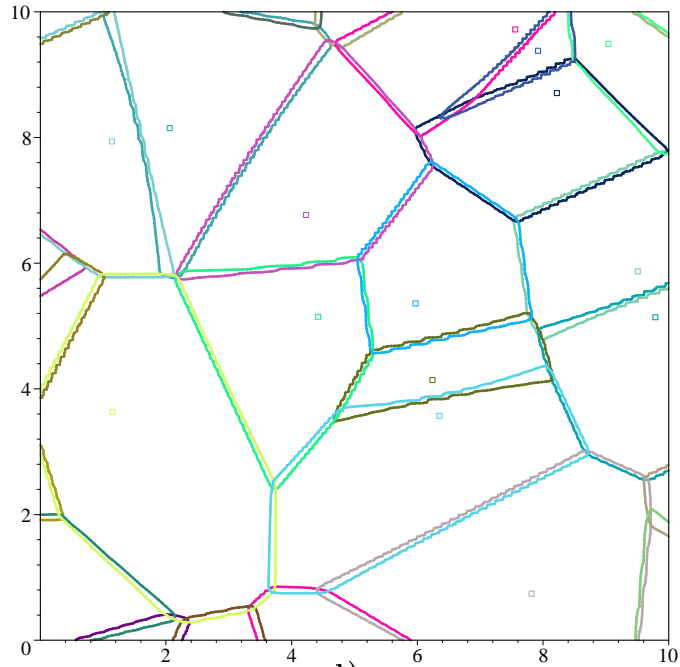

d)

Figure 4: The first coverage process of Example 6.17 tending to the Voronoi tessellation of the plane .

than

$$
\begin{aligned}
|y-X| & \leq\left(\frac{S}{b I_{\Phi}(y)+c}\right)^{1 / 30}-1 \\
& \leq\left(\frac{S}{c}\right)^{1 / 30}-1 \\
& =b^{-1 / 30} \frac{1+R-1.2}{(1+R)(c / b)^{1 / 30}}-1 \\
& =b^{-1 / 30}(R-0.2)-1 \approx R-1.2,
\end{aligned}
$$

that is, the new cell is always "younger" (its maximal diameter is less) than others. Note however that, unlike in the original Johnson-Mehl model, the younger cell can conquer the space already taken by an older cell. Thus in d) we have almost the Voronoi tessellation, where no effect of coming late can be detected. 


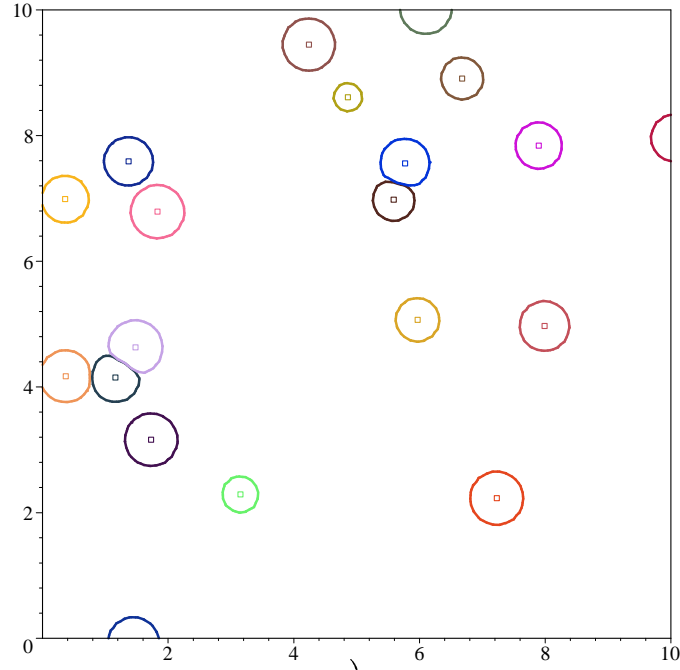

a)

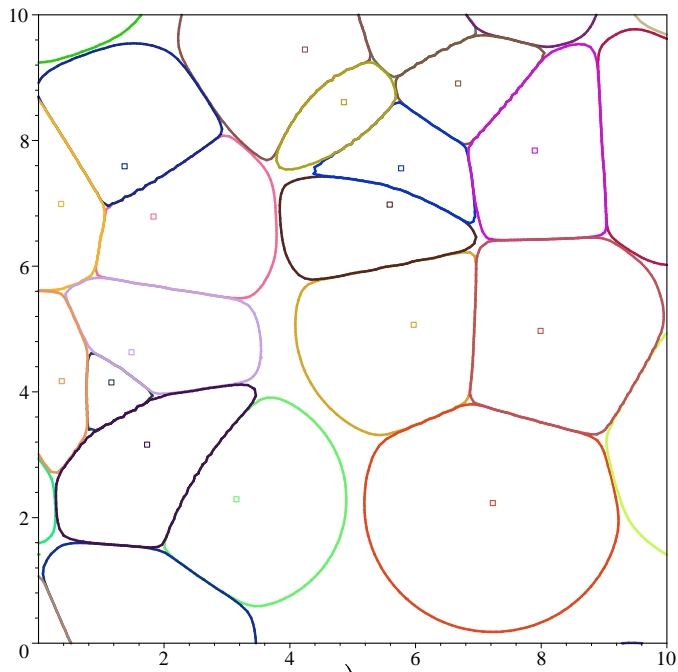

c)

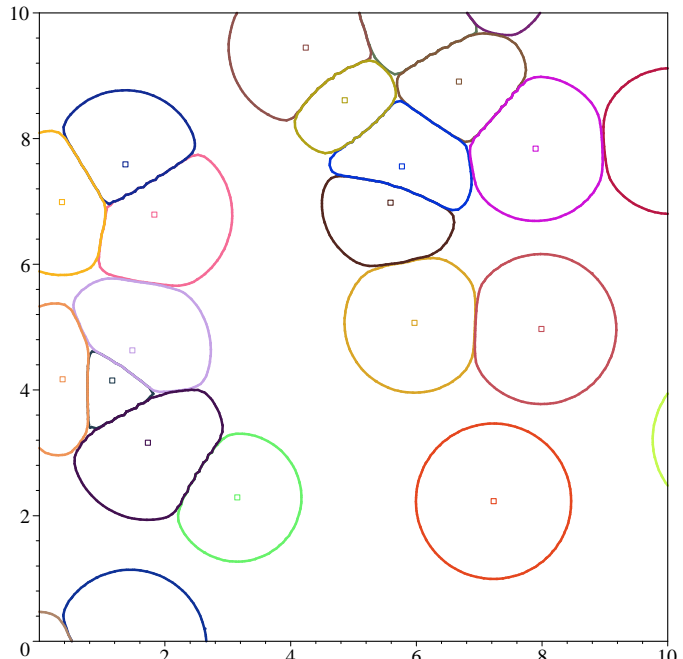

b)

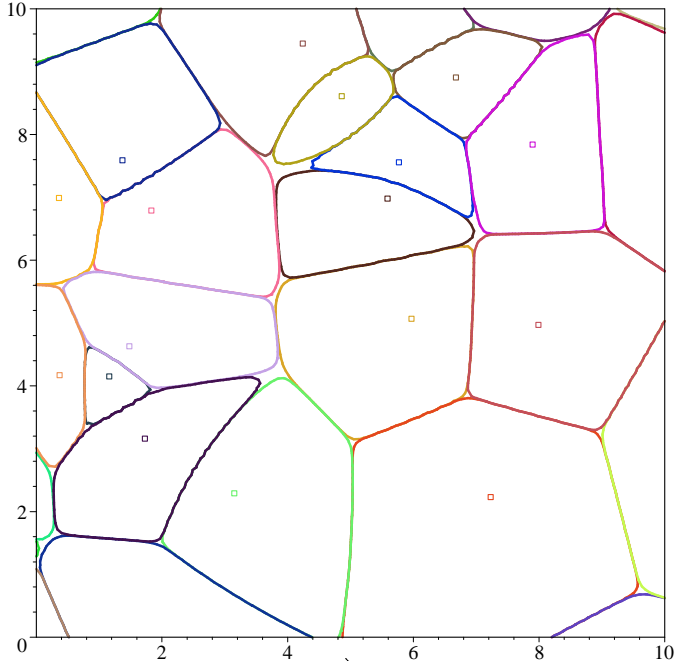

d)

Figure 5: The coverage process of Example 6.19 growing as in the Johnson-Mehl model to the Voronoi tessellation of the plane.

\section{Appendix}

In the following proposition we show how to find the probability for an absolutely continuous real valued random variable $Y$ to be nonnegative knowing its Fourier transform. We explain how to reduce this to the solution of a Reimann boundary problem (see [6]) on the real line and show that the solution of this problem given in terms of a singular integral on the real line.

Proposition A.1 Suppose that the real valued random variable $Y$ has a density and denote by $\psi(\xi)=\mathrm{E}[\exp (-i \xi Y)], \xi \in \mathbb{R}$, its Fourier transform. Then

$$
\mathrm{P}(Y \geq 0)=\frac{1}{2}-\frac{1}{2 i \pi} \int_{\mathbb{R}} \frac{\psi(\xi)}{\xi} d \xi
$$

where the singular contour integral in the right hand side is understood in the principal value sense. 


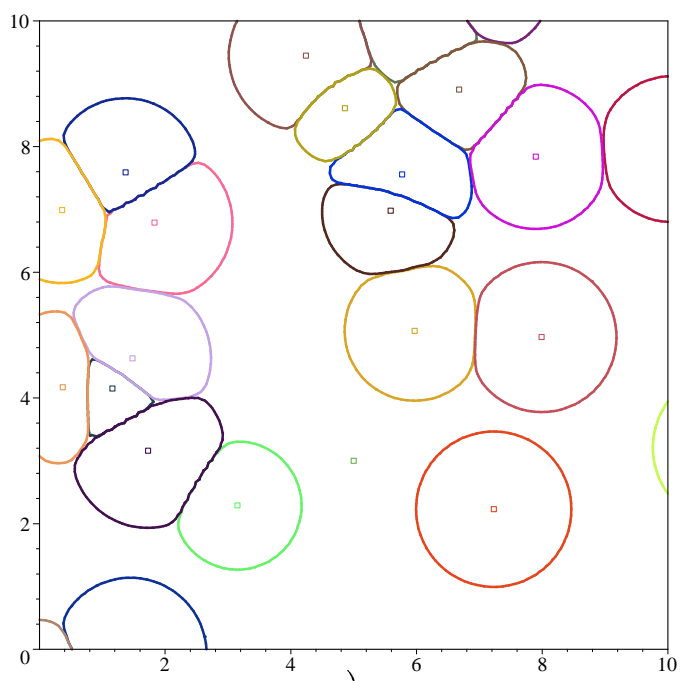

a)

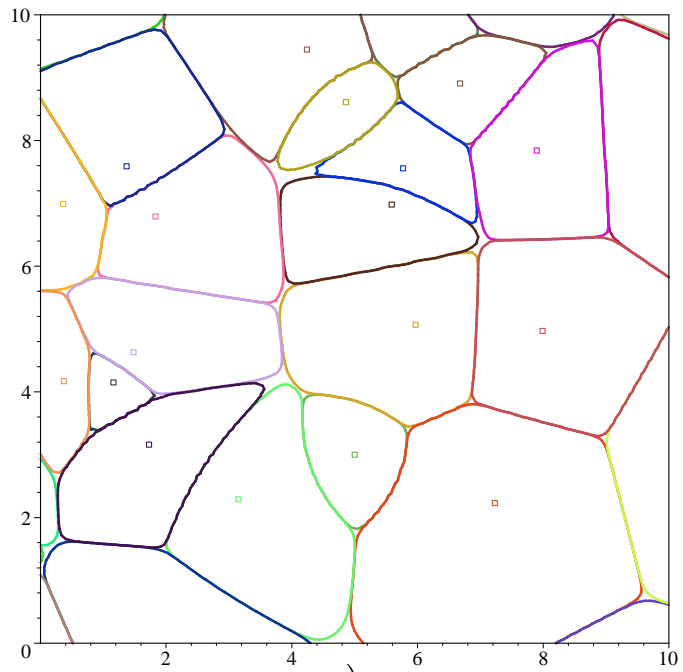

c)

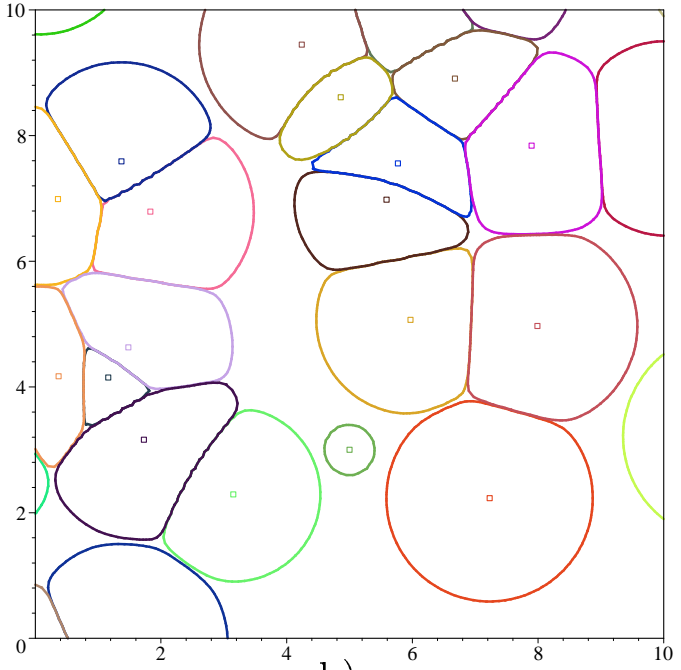

b)

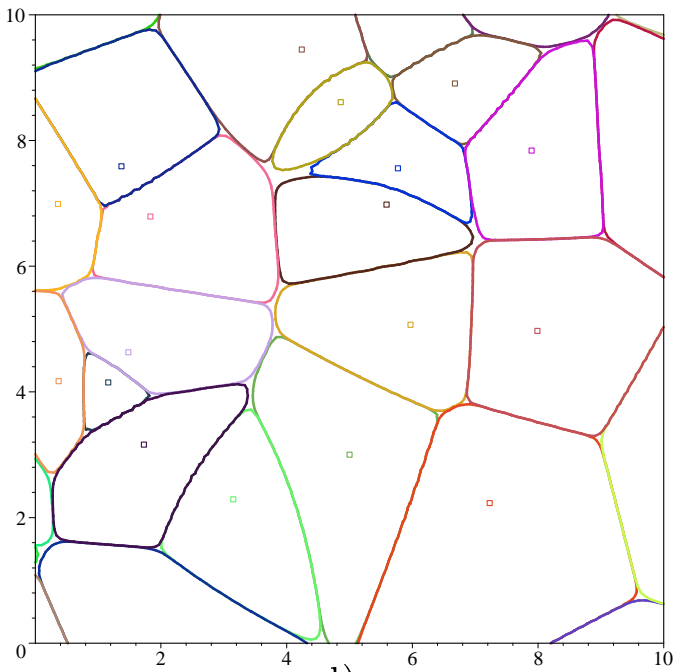

d)

Figure 6: The late comer of Example 6.19.

Proof: Denote the density of $Y$ by $f(x)$. If we define $f^{+}(x)=f(x) 1_{x \geq 0}$ and $f^{-}(x)=f(x) 1_{x<0}$, then

$$
f(x)=f^{+}(x)+f^{-}(x), \quad \text { for } x \in \mathbb{R}
$$

and the probability of interest, $\mathrm{P}(Y \geq 0)$, is the integral of $f^{+}(x)$ over $\mathbb{R}$.

Let us define the function $\Psi(z)$ from the imaginary axis to the complez plane by $\Psi(i \xi)=$ $\psi(\xi), \xi \in \mathbb{R}$. Because $\psi(\xi)$ is the Fourier transform of an absolutely continuous random variable the following two properties hold:

1. the function $\Psi(z)$ satisfies Hölder condition on the imaginary axis (cf. e.g. [5], Lemma 3, p. 513);

2. $\Psi(z)$ is integrable on the imaginary axis (cf. e.g. [5], the corollary of Theorem 3, p. 510), which implies that $\Psi(z) \rightarrow 0$ when $|z| \rightarrow \infty$ and $|\Psi(z)| \leq A /|z|$ for some $A$ and for large $|z|$. 
Let us define the two functions $\Psi^{+}(z)=\int_{-\infty}^{\infty} e^{-z x} f^{+}(x) d x$ for $\operatorname{Re}(z) \geq 0(\operatorname{Re}(z)$ denotes the real part of the complex number $z$ ) and $\Psi^{-}(z)=\int_{-\infty}^{\infty} e^{-z x} f^{-}(x) d x$ for $\operatorname{Re}(z) \leq 0$. Note that they are continuous in their domains and analytic in $\operatorname{Re}(z)>0$ and $\operatorname{Re}(z)<0$, respectively, and that both vanish at infinity. From (A.2) we see that they satisfy

$$
\Psi(z)=\Psi^{+}(z)+\Psi^{-}(z)
$$

for $z$ on the imaginary axis. This means the pair $\Psi^{+}(z), \Psi^{-}(z)$ is a solution of a so called Riemann boundary value problem posed on the imaginary axis (see [6], Chapter 2). Under the conditions 1 and 2 mentioned above, the solution of this boundary value problem is unique and can be obtained via Sokhotski's formulas, in particular, when $\psi(0)=1, \Psi^{+}(0)$ is equal to the right hand side of (A.1) (see §4.6, §14.2 and following in [6]). On the other hand, by our definition, $\Psi^{+}(0)=\mathrm{P}(Y \geq 0)$.

Remark: Note that the function to be integrated in (A.1) has a pole at $\xi=0$. In order to calculate this so called singular integral in the principal value sense, one has to calculate the integral over the domain $(-\infty,-\epsilon] \cup[\epsilon, \infty)$ and then let $\epsilon$ decrease to 0 (see [6]).

In the following proposition we give simple conditions for the Poisson shot-noise random variable $I_{\Phi}(y)$ to be absolutely continuous with respect to Lebesgue measure.

Proposition A.2 For any $y \in \mathbb{R}^{d}$, the random variable $I_{\Phi}(y)$ is absolutely continuous with respect to the Lebesgue measure (has a density) if $\mu(\mathbb{R})=\infty$ and for each $A \subset \mathbb{R}^{+}$of Lebesgue measure 0 ,

$$
\int_{\mathbb{R}^{d} \times \mathbb{D}} \mathbb{I}(L(s, x) \in A) \mu(d x) H(d s)=0
$$

Proof: Fix $y$, without loss of generality let $y=0$. Take $A \subset \mathbb{R}_{+}$of Lebesgue measure 0 . Then for any $r>0$

$$
\mathrm{P}\left(I_{\Phi}(0) \in A\right)=\mathrm{P}\left(I_{r}+I_{r}^{c} \in A\right),
$$

where $I_{r}=\int_{B(0, r) \times \mathbb{D}} L(s,-x) \Phi(d(x, s))$ and $I_{r}^{c}=\int_{\left(\mathbb{R}^{d} \backslash B(0, r)\right) \times \mathbb{D}} L(s,-x) \Phi(d(x, s))$. By the Poisson assumption $I_{r}$ and $I_{r}^{c}$ are independent. Moreover

$$
\mathrm{P}\left(I_{\Phi}(0) \in A\right)=\sum_{n=0}^{\infty} \mathrm{P}\left(I_{r}+I_{r}^{c} \in A \mid \Phi(B(0, r) \times \mathbb{D})=n\right) \mathrm{P}(\Phi(B(0, r) \times \mathbb{D})=n) .
$$

Note that conditioned on $\Phi(B(0, r) \times \mathbb{D})=n$, with $n>0$, the random variable $I_{r}$ can be represented as the sum on $n$ independent random variables, distributed as $L(S,-X)$ where $S$ and $X$ are independent, with distributions $H(d s)$ and $\mu(d x) / \mu(B(0, r)) \mathbb{I}(x \in B(0, r))$ respectively. By (A.3) $\mathrm{P}\left(I_{r}+I_{r}^{c} \in A \mid \Phi\left(B(0, r) \times \mathbb{R}_{+}\right)=n\right)=0$. Thus

$$
\mathrm{P}\left(I_{\Phi}(0) \in A\right) \leq \mathrm{P}\left(\Phi\left(B(0, r) \times \mathbb{R}_{+}\right)=0\right) \rightarrow 0 \quad \text { when } r \rightarrow \infty
$$




\section{References}

[1] Baccelli, F., Klein, M., Lebourges, M., and Zuyev, S., (1997), Stochastic geometry and architecture of communication networks. Telecommunications systems 7, pp. 209-227.

[2] Błaszczyszyn, B., Merzbach, E. and Schmidt, V. (1997), A note on expansion for functionals of spatial marked point processes. Statist. and Probab. Lett. 36, 299-306.

[3] Buldygin, V. and Yarovaya, N.V. (1983) A functional limit theorem for shot noise fields. (in Russian) Problems of the theory of probability distributions 25-41, Akad. Nauk Ukrain. SSR, Inst. Mat., Kiev.

[4] Ehrenberger, U. and Leibnitz, K. (1999) Impact of clustered traffic distributions in CDMA radio network planning, in Teletraffic Engineering in a Competitive World by Key, P. and Smith, D. (editors) Elsevier, Amsterdam, 129-138.

[5] Feller, W. (1971) An Introduction to Probability Theory and its Applications, vol. II, J. Wiley \& Sons, New York.

[6] Gakhov, F.D. (1990), Boundary Value Problems Dover.

[7] Gubner, J. (1996) Computation of shot-noise probability distributions and densities. SIAM J. Comput. 17, 750-761.

[8] Gupta, P. and Kumar, P.R. (2000), The Capacity of Wireless Networks, IEEE Transactions on Information Theory, pp. 388-404, vol. IT-46, no. 2, March 2000.

[9] Hall, P. (1988), Introduction to the Theory of Coverage Processes. J. Wiley \& Sons, New York.

[10] Heinrich, L. and Molchanov, I.S. (1994) Some limit theorems for extremal and union shotnoise processes. Math. Nach. 168, 139-159.

[11] Heinrich, L. and Schmidt, V. (1985) Normal convergence of multidimensional shot noise and rates of this convergence. Adv. in Appl. Probab. 17, 709-730.

[12] Matheron, G. (1975) Random Sets and Integral Geometry, John Wiley \& Sons, London.

[13] Orsingher, E. and Battaglia, F. (1982/83) Probability distributions of level crossings of shot noise models. Stochastics 8, 45-61.

[14] Rice, J. (1977) On a generalized shot noise. Adv. in Appl. Probab. 17, 709-730.

[15] Schmidt, V. (1985) On finiteness and continuity of shot-noise process. Optimization, 16, 921-933.

[16] Schmidt, V. (1987) Qualitative and asymptotic properties of stochastic integrals related to random marked point processes. Optimization, 18, 737-759.

[17] Stoyan, D., Kendall, W and Mecke, J. (1995) Stochastic Geometry and its Applications, John Willey \& Sons, Chichester. 
[18] Westcott, M. (1976) On the existence of a generalized shot-noise process. In Williams, E.J., editor, Studies in Probability and Statistics. Papers in Honour of Edwin J.G. Pitman, North-Holland, Amsterdam, pages 73-88.

[19] Veeravalli, V. and Sendonaris, A. (1999) The Coverage-Capacity Tradeoff in Cellular CDMA Systems." IEEE Transactions on Vehicular Technology 48 1443-1451 


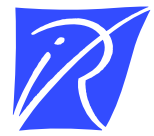

Unité de recherche INRIA Rocquencourt Domaine de Voluceau - Rocquencourt - B.P. 105 - 78153 Le Chesnay Cedex (France)

Unité de recherche INRIA Lorraine : Technopôle de Nancy-Brabois - Campus scientifique 615, rue du Jardin Botanique - B.P. 101 - 54602 Villers lès Nancy Cedex (France)

Unité de recherche INRIA Rennes : IRISA, Campus universitaire de Beaulieu - 35042 Rennes Cedex (France)

Unité de recherche INRIA Rhône-Alpes : 655, avenue de l'Europe - 38330 Montbonnot St Martin (France)

Unité de recherche INRIA Sophia Antipolis : 2004, route des Lucioles - B.P. 93 - 06902 Sophia Antipolis Cedex (France)

INRIA - Domaine de Voluceau - Rocquencourt, B.P. 105 - 78153 Le Chesnay Cedex (France)

http://www.inria.fr

ISSN 0249-6399 